\title{
Deficient adaptation to centrosome duplication defects in neural progenitors causes microcephaly and subcortical heterotopias
}

José González-Martínez, ${ }^{1}$ Andrzej W. Cwetsch, ${ }^{1,2,3}$ Diego Martínez-Alonso, ${ }^{1}$ Luis R. López-Sainz, ${ }^{1}$ Jorge Almagro, ${ }^{4}$ Anna Melati, ${ }^{1}$ Jesús Gómez, ${ }^{5}$ Manuel Pérez-Martínez, ${ }^{5}$ Diego Megías, ${ }^{5}$ Jasminka Boskovic, ${ }^{6}$ Javier Gilabert-Juan, ${ }^{1,7}$ Osvaldo Graña-Castro, ${ }^{8}$ Alessandra Pierani, ${ }^{2,3}$ Axel Behrens, ${ }^{4,9}$ Sagrario Ortega, ${ }^{10}$ and Marcos Malumbres ${ }^{1}$

${ }^{1}$ Cell Division and Cancer group, Spanish National Cancer Research Centre (CNIO), Madrid, Spain. ${ }^{2}$ Imagine Institute of Genetic Diseases, University of Paris, Paris, France. ${ }^{3}$ Institute of Psychiatry and Neuroscience of Paris, INSERM U-1266, University of Paris, Paris, France. ${ }^{4}$ Adult Stem Cell Laboratory, The Francis Crick Institute, London, United Kingdom. ${ }^{5}$ Confocal Microscopy Unit and ${ }^{6}$ Electron Microscopy Unit, CNIO, Madrid, Spain. 'University of Paris, NeuroDiderot, Inserm, Paris, France. ${ }^{8}$ Bioinformatics Unit, CNIO, Madrid, Spain. ${ }^{9}$ Faculty of Life Sciences, King's College London, Guy's Campus, London, United Kingdom. ${ }^{10}$ Mouse Gene Editing Unit, CNIO, Madrid, Spain.

Congenital microcephaly (MCPH) is a neurodevelopmental disease associated with mutations in genes encoding proteins involved in centrosomal and chromosomal dynamics during mitosis. Detailed MCPH pathogenesis at the cellular level is still elusive, given the diversity of $M C P H$ genes and lack of comparative in vivo studies. By generating a series of CRISPR/Cas9-mediated genetic $\mathrm{KOs}$, we report here that - whereas defects in spindle pole proteins (ASPM, MCPH5) result in mild MCPH during development - lack of centrosome (CDK5RAP2, MCPH3) or centriole (CEP135, $M C P H 8$ ) regulators induces delayed chromosome segregation and chromosomal instability in neural progenitors (NPs). Our mouse model of MCPH8 suggests that loss of CEP135 results in centriole duplication defects, TP53 activation, and cell death of NPs. Trp53 ablation in a Cep135deficient background prevents cell death but not MCPH, and it leads to subcortical heterotopias, a malformation seen in MCPH8 patients. These results suggest that MCPH in some MCPH patients can arise from the lack of adaptation to centriole defects in NPs and may lead to architectural defects if chromosomally unstable cells are not eliminated during brain development.

Authorship note: AWC, DMA, and LRLS contributed equally to this work.

Conflict of interest: The authors have declared that no conflict of interest exists.

Copyright: (c) 2021, GonzálezMartínez et al. This is an open access article published under the terms of the Creative Commons Attribution 4.0 International License.

Submitted: November 24, 2020

Accepted: July 7, 2021

Published: August 23, 2021

Reference information: /CI Insight. 2021;6(16):e146364.

https://doi.org/10.1172/jici. insight.146364.

\section{Introduction}

Autosomal recessive primary microcephaly $(\mathrm{MCPH})$ is a congenital brain disorder characterized by a reduction in head circumference linked to a striking decrease in brain volume (from -3 to -13 SDs). These changes are typically not linked with gross anomalies of brain architecture and associate with a primary and selective defect in the production of neurons during development. Several genes mutated in $\mathrm{MCPH}$ patients have been identified so far (MCPH1-25), including genes encoding proteins associated with centriole biology or the mitotic spindle such as ASPM (MCPH5) and WDR62 (MCPH2), the 2 most commonly mutated MCPH genes (1-4) (Figure 1A). Centrioles are barrel-shaped, membrane-less organelles that behave as the major microtubule organizing centers (MTOCs) generating key cellular structures such as centrosomes, cilia, and flagella. Structurally, centrioles consist of 9 microtubule triplets organized around a cartwheel from which 9 protein spokes emanate radially, conferring a 9-fold symmetric conformation that stabilizes the structure (5). These structures recruit several proteins to polymerize a robust pericentriolar matrix (PCM), thereby allowing the generation of a bipolar spindle that ensures the correct segregation of chromosomes to both daughter cells, thus avoiding chromosomal instability. Deregulation of centrosome dynamics typically results in a variety of cell division defects, ultimately affecting neural development (4).

Centriole duplication relies on the formation of a newborn procentriole in an orthogonal angle using the preexisting centriole as a biogenesis platform (5). The core centriole duplication toolbox required for 
A

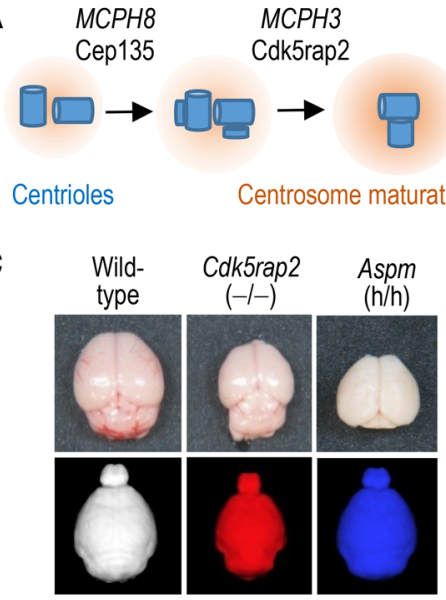

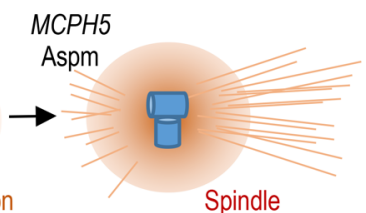

B

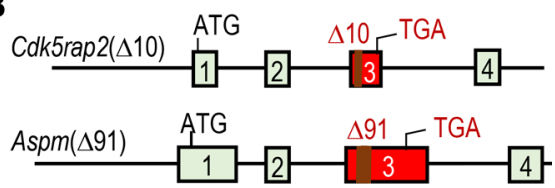

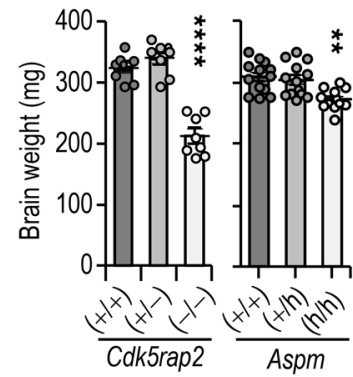
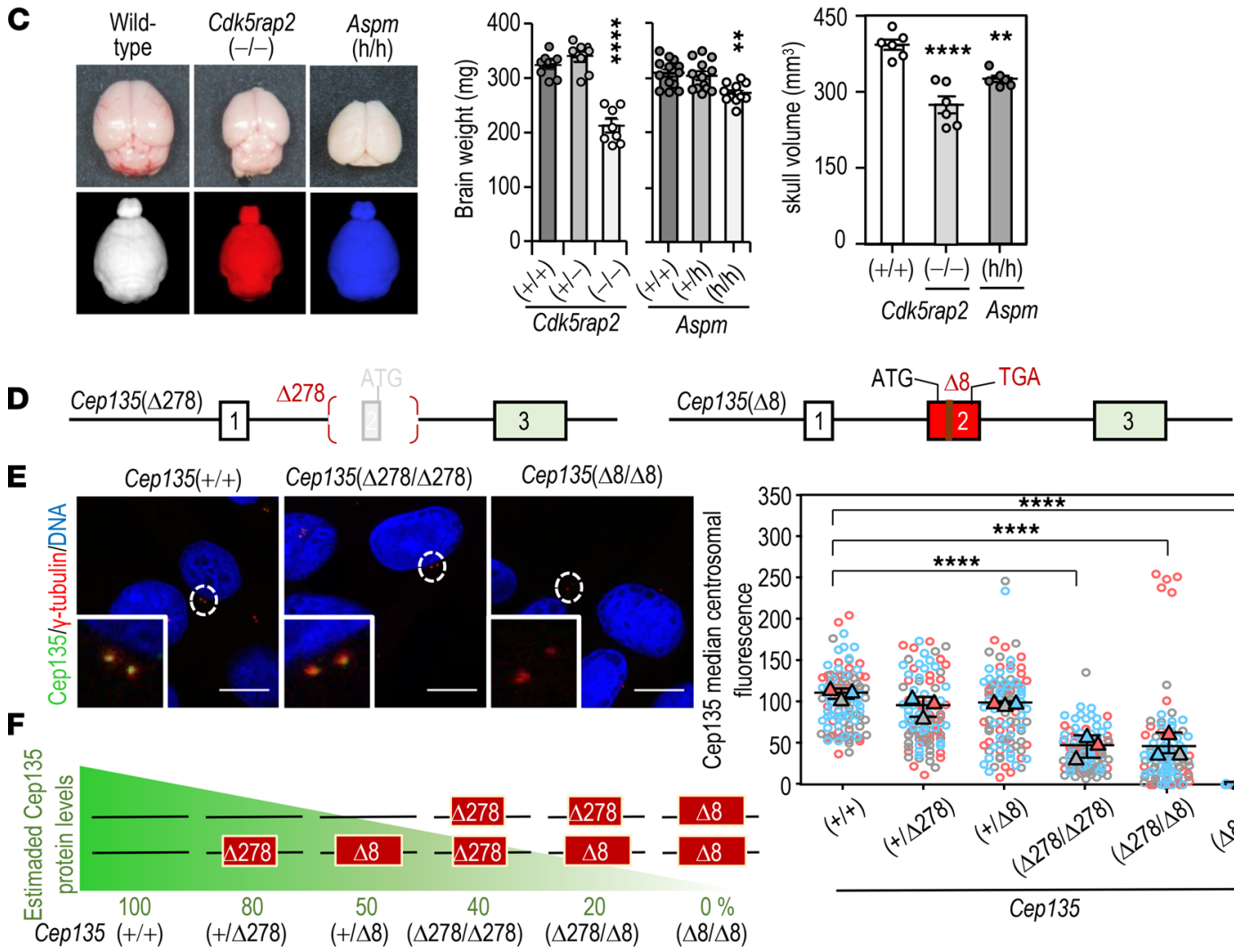

E $\begin{array}{llllll}\text { Cep135 }(+/+) \quad(+/ \Delta 278) \quad(+/ \Delta 8) & (\Delta 278 / \Delta 278) & (\Delta 278 / \Delta 8) & (\Delta 8 / \Delta 8)\end{array}$
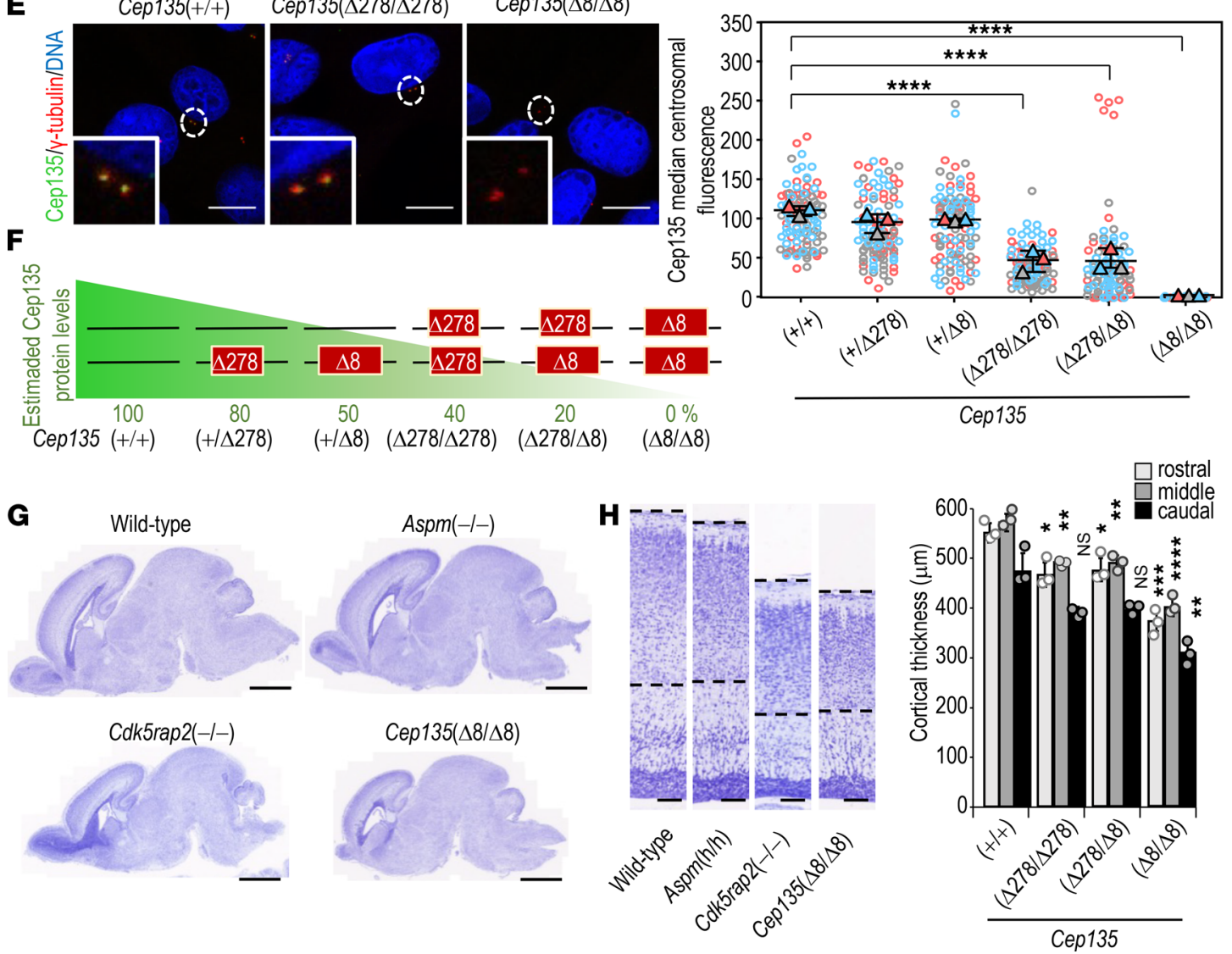

Figure 1. A comparative analysis of MCPH models in the mouse. (A) Role of MCPH8 proteins in centriole duplication, centrosome maturation, and spindle

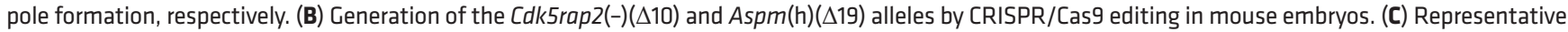
images of brains derived from P30 mice (upper) and skull volumes as scored by CT-scan analysis (lower). Histograms to the right depict the quantifications of brain weight of P30 Cdk5rap2-mutant $(n=8)$ and Aspm-mutant $(n=12)$ mice and the skull volume at P30 ( $n=6 /$ group). (D) Generation of the Cep135( $\Delta 278)$ and Cep135( $\Delta 8)$ alleles. The Cep135( $\Delta 278)$ allele lacks the initial ATC site. The $\Delta 8$ allele generates of a premature stop codon in exon 3 . (E) Confocal imaging of centriolar loading of CEP135 (green) in MEFs with different combinations of Cep135( $\Delta 278)$-mutant and Cep135( $\Delta 8)$-mutant alleles. $\gamma$-Tubulin, red; CEP135, green; DNA, blue. Stained with DAPI. Scale bars: $10 \mu \mathrm{m}$. The superplot to the right shows the quantification of CEP135 centrosomal protein levels in cells with the indicated genotypes. Each color represents data from a different mouse. Horizontal bars depict the mean; ${ }^{* * *} P<0.001$ (unpaired $t$ test with Welsh correction). (F) A summary of the effect of the different Cep135 alleles in the centrosomal levels of CEP135. (G) Representative micrographs of sagittal brain sections stained with Nissl at PO in neonate Aspm, Cdk5rap2, and Cep135 mutants. Scale bars: 1 mm. (H) Histological Nissl staining of PO cortices from the indicated mice (left panels). Scale bars: $100 \mu \mathrm{m}$. Quantification of cortical thickness in rostral, medial, and caudal aspects of P0 Cep135-mutant mice versus Cep135(+/+) controls (histogram to the right). Data in $\mathbf{C}$ and $\mathbf{H}$ represent mean \pm SEM from 3 different mice or embryos; ${ }^{*} P<0.05 ;{ }^{* *} P<0.01 ;{ }^{* * *} P<0.001 ;{ }^{* * *} P<0.0001$; 1-way ANOVA with Tukey's multiple comparisons. 
this process includes enzymatic components such as Polo-like kinase 4 (PLK4), which acts as a key regulator of centriole biogenesis $(6,7)$, as well as structural components such as SAS4 (MCPH6, also known as CPAP or CENPJ) (8), SAS6 (MCPH14) (9), or CEP135 (MCPH8) (10). CEP135 was originally identified as a component of the cartwheel, with critical scaffold function in centriole structure and biogenesis (10). Pioneer studies in Drosophila suggested minor defects in centriole duplication after Cep135 knockdown (11-14), as well as mild perturbations of centriole structure in Cep135-mutant spermatocytes (14). Cep135 knockdown in chicken cell lines does not significantly alter centriole structure and organization, nor centriole biogenesis or cell proliferation, and only mild centrosome duplication defects arise (15). In contrast, genetic knockdown by RNA interference of CEP135 in human cell lines induced significant centriole defects, altered spindle assembly and aberrant centriole structure (16). Interestingly, genetic analysis of a cohort of $\mathrm{MCPH}$ patients identified a homozygous single bp deletion in exon 8 of human CEP135, which resulted in a frameshift that produced a premature stop codon (17). Patients bearing this mutation present severe $\mathrm{MCPH}$ accompanied by subcortical heterotopia (cell clumps between the lateral ventricles and the cortex; ref. 18) - a finding not common in other $\mathrm{MCPH}$ patients and whose pathogenic origin is unclear.

In this work, we have generated a series of $\mathrm{MCPH}$ models with specific alterations in centriole (CEP135), centrosome (CDK5RAP2), and spindle pole (ASPM) proteins by using CRISPR/Cas9-mediated direct editing of mouse embryos. Whereas Aspm ablation results in minor defects in cortical development, lack of Cdk5rap2 or Cep135 results in significant primary $\mathrm{MCPH}$ accompanied by centrosome duplication, chromosomal instability, and a TP53-associated antiproliferative response in the developmental cortex. Genetic ablation of TP53 prevents apoptosis during midgestation but does not prevent MCPH and leads to heterotopia in late development, a relatively rare finding in $\mathrm{MCPH}$ (including $\mathrm{MCPH}$ ), as well as in patients with seizures and intellectual disability.

\section{Results}

A comparative analysis of MCPH models in the mouse. We first generated mouse models of MCPH3 and MCPH5 (Figure 1A) by introducing loss-of-function mutations in the murine Cdk5rap2 and Aspm genes (Figure 1B). In agreement with previous mouse models for these pathologies (19-21), lack of Cdk5rap2 (Cdk5rap2[-] allele) and partial lack of Aspm (Aspm[h] allele) (Supplemental Figure 1A; supplemental material available online with this article; https://doi.org/10.1172/jci.insight.146364DS1) resulted in significant defects in brain weight and volume at P30 (Figure 1C and Supplemental Figure 1B). Both Cdk5rap2(-/-) and Aspm(h/h) mice displayed reduced maturation of germ cells in the testis (Supplemental Figure 1, C and D) as reported previously $(19,20)$. We also observed a previously undefined defect in retinal development causing microphtalmia in Cdk5rap2-deficient embryos (Supplemental Figure 1E), in agreement with cataracts and ocular abnormalities observed in MCPH3 patients $(22,23)$. Cortical thickness was reduced in both models at E14.5 and adult (P30) neocortices, with a stronger phenotype in the absence of Cdk5rap2 (Supplemental Figure 1, F and G).

Since a previous model of MCPH8 did not exist, we next decided to generate mice with loss-of-function mutations in Cep135. We targeted the second exon of murine Cep135 using 2 single-guide RNAs (sgRNAs) generating a mutant sequence with a 278 bp deletion (Cep135[ $\Delta 278]$ allele lacking the initial ATG site) and an 8 bp deletion (Cep135[ $\Delta 8]$ allele) (Figure 1D and Supplemental Figure 2A). Cep135( $\Delta 278 /$ $\triangle 278$ ) mouse embryonic fibroblasts (MEFs) displayed reduced protein levels at the centrosome, whereas no detectable CEP135 was present in Cep135( $\Delta 8 / \Delta 8)$ cells (Figure 1E). Combination of these alleles in either homozygosity or heterozygosity allowed the generation of 6 cohorts of Cep135-mutant mice with different amounts of CEP135 protein, ranging from WT levels to intermediate levels in Cep135( $\Delta 278$ / $\Delta 278)$ homozygous mutants and $C \operatorname{ep} 135(\Delta 278 / \Delta 8)$ heterozygous mice, and no detectable protein in the Cep135( $\Delta 8 / \Delta 8)$ model (Figure $1 \mathrm{~F})$.

These alleles allowed a comparison between Cep135 gene dosage and the phenotype arising from different CEP135 protein levels in vivo. Both Cep135( $\Delta 278 / \Delta 278)$ and $C e p 135(\Delta 278 / \Delta 8)$ newborns $(\mathrm{P} 0)$ displayed mild MCPH ( $\sim 24.5 \%$ and $24.7 \%$ reduction in cortical area, respectively), whereas homozygous $C e p 135(\Delta 8 /$ $\triangle 8)$ P0 mice showed severe MCPH, as showed by a reduced neocortical area (48.5\% cortical area reduction; Supplemental Figure 2, B-E, and Figure 1, G and H) and reduced neocortical thickness in the rostral, medial, and caudal aspects of the neonatal neocortices (Figure 1G). The reduction in head and brain weight was more pronounced than the reduction in body weight (Supplemental Figure 2E), suggesting that Cep135 depletion may promote $\mathrm{MCPH}$ in a gene dose-dependent manner in mice. Full depletion of CEP135 in the Cep135( $\Delta 8 /$ $\Delta 8$ ) model (Figure 1E) resulted in perinatal lethality, with all mutant mice dying within a few hours after 
birth. Histological examination of Cep135-null i.p. organs revealed simplified and underdeveloped intestinal and stomach mucosae, with no evidence of recent lactation, immature and underdeveloped lungs, and retinal abnormalities (Supplemental Figure 2, F and G). Detailed analysis of the lungs revealed small, immature alveolae with lower levels of Surfactant Protein-C (Supplemental Figure 2H), suggestive of respiratory distress. Cep135(+/ $\Delta 278)$ and $C e p 135(+/ \Delta 8)$ heterozygous mice exhibited no significant differences in the centrosomal levels of CEP135 (Figure 1E) and, accordingly, no $\mathrm{MCPH}$, body size reduction, or other phenotypic defects were observed (data not shown).

Lack of Cep135 induces multiple defects in centriole dynamics and chromosomal instability. To gain a deeper insight about the cellular alterations generated by CEP135 loss, we initially screened for centrosome or cytoskeleton defects in CEP135-deficient MEFs. Whereas 2 foci of $\gamma$-tubulin (a major component of the pericentriolar material $[\mathrm{PCM}]$ ) were detected in most control cells positive for cyclin A (a marker of late S-G2 phases of the cell cycle), only $1 \gamma$-tubulin structure was found in the majority of cyclin $\mathrm{A}^{+}$cells derived from Cep135-KO embryos (Figure 2A). For a more detailed analysis of cell cycle stages, we made use of proliferating cell nuclear antigen (PCNA), a DNA replication processivity factor whose nuclear pattern changes depending of the period of the S-phase, and phospho-histone $\mathrm{H} 3$ (PH3), a modification that correlates with chromosome condensation during late G2 and mitosis. Whereas both control and Cep135-mutant cells displayed a single centrosome in G1, coimmunostaining of $\gamma$-tubulin with PCNA revealed efficient centrosome duplication in control Cep135(+/+) but not in mutant $C$ ep 135( $\Delta 8 / \Delta 8$ ) cells (Figure 2B). In about 50\% of Cep135-null cells, a single $\gamma$-tubulin spot was present from G1 to late G2, inducing monopolar spindles during mitosis (Figure 2B). Interestingly, the remaining fraction of Cep135-KO cells that contained 2 centrosomes presented additional defects, such as centrosomal asymmetry in the maturation of centrosomes when stained for Centrin (a centriolar protein) and the PCM component $\gamma$-tubulin. This defect typically affected the daughter, newly generated centrosome, and not the mother centrosome, which is characterized by the presence of Outer Dense Fiber Protein 2 (ODF2; Figure 2C).

Combined immunofluorescence for centrin and $\gamma$-tubulin in cyclin A-positive cells indicated that centrosome duplication was compromised in $C e p 135(\Delta 8 / \Delta 8)$ cells (Figure 2D, group V). In addition, these mutant cultures were enriched in cells with only 1 centrosome and 1 centriole, suggestive of both centrosome duplication and centriole assembly defects (Figure 2D, group VI), as well as in cells lacking centrosomes (acentrosomal, group VII). Similar results were obtained in late $\mathrm{G} 2 \mathrm{PH}^{+}$cells, confirming that centriole defects caused by CEP135 depletion were also sustained during the rest of the cell cycle (Supplemental Figure 3A). Immunostaining of S/G2 cells (Cyclin $\mathrm{A}^{+}$) for PCM ( $\gamma$-tubulin) and cartwheel proteins (Sas6) revealed a significant increase in cells with only 1 centrosome devoid of cartwheel (Figure 2E; group V), suggesting additional defects in cartwheel assembly. Interestingly, MEFs derived from Aspm- and Cdk5rap2-mutant embryos did not present significant centrosome duplication defects (Figure $2 \mathrm{~F}$ ), suggesting that centrosome duplication defects are a specific feature generated by the absence of CEP135.

The presence of 2 properly matured and separated centrosomes is critical for the establishment of a bipolar spindle and correct chromosome segregation. Time-lapse microscopy analysis of $\operatorname{Cep} 135(\Delta 278 /$ $\Delta 278)$ or Cep135( $\Delta 278 / \Delta 8)$ cultures showed no alteration in the duration of mitosis. However, homozygous cells for the Cep135( $\Delta 8)$ mutation displayed significantly increased duration of mitosis, in agreement with abundant monopolar spindles (Supplemental Figure 3, B and C). These defects were accompanied by increased nuclear volume, DNA content ( $4 \mathrm{n}$ and more than $4 \mathrm{n}$ ) and aneuploidy in a Cep135 gene dose-dependent manner (Supplemental Figure 3, D-F). These results suggest that loss of CEP135 in the Cep135( $\Delta 8 / \Delta 8)$ model leads to defective centriole dynamics, ultimately inducing chromosomal instability, at least in fibroblasts.

Perturbed centrosomal and mitotic dynamics in Cep135-deficient neural progenitors. We next examined the effect of lack of CEP135 in cortical neural progenitors (NPs) isolated from E14.5 neocortices and cultured to generate incipient neurospheres. In agreement with the observations in fibroblasts, Cep135-deficient neurospheres displayed centrosome duplication defects, whereas this effect was not evident in Cdk5rap2- or Aspm-deficient progenitors (Figure 3A). Lack of Cep135 also correlated with a significant defect in their self-renewal capacity, as scored by the limiting dilution assays (Figure $3 \mathrm{~B}$ ). Unfortunately, these defects and the reduced viability of these mutant cells (see below) did not allow to establish cultures of $\operatorname{Cep} 135(\Delta 8 / \Delta 8)$ NPs for further assays of centrosome dynamics in vitro.

We therefore looked for centrosome alterations in vivo. The reduced cortical thickness observed at birth (Figure 1) was also present in these mutant mice at E14.5 and E17.5, and it specifically affected the SOX2 ${ }^{+}$ 
A

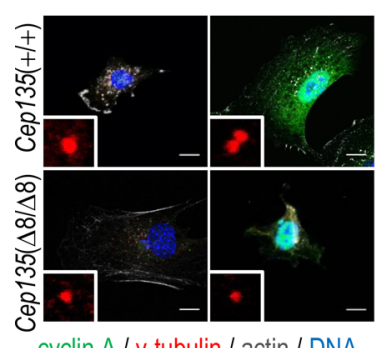

cyclin A / p-tubulin / actin / DNA
B

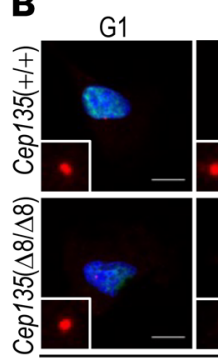

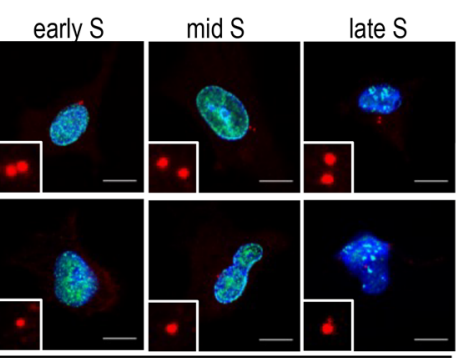

PCNA / y-tubulin / DNA
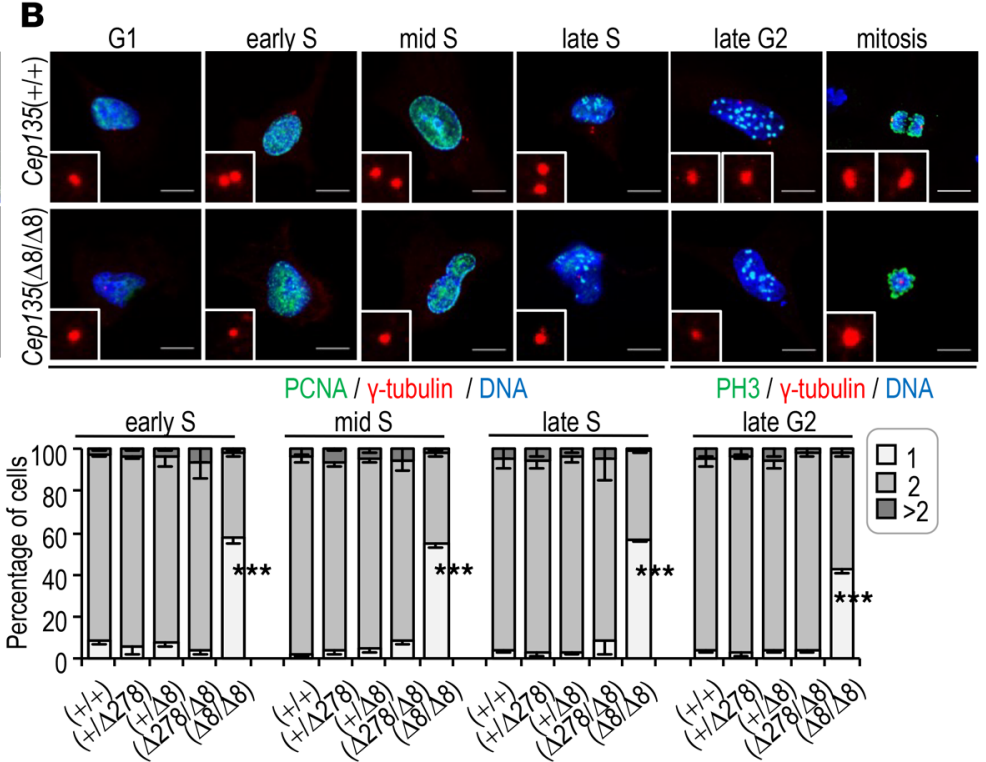

PH3 / y-tubulin / DNA

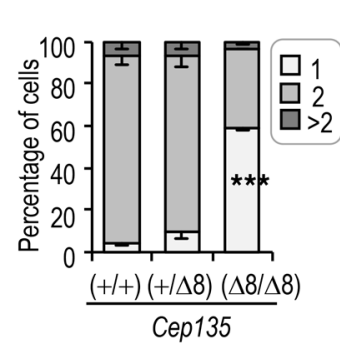

D
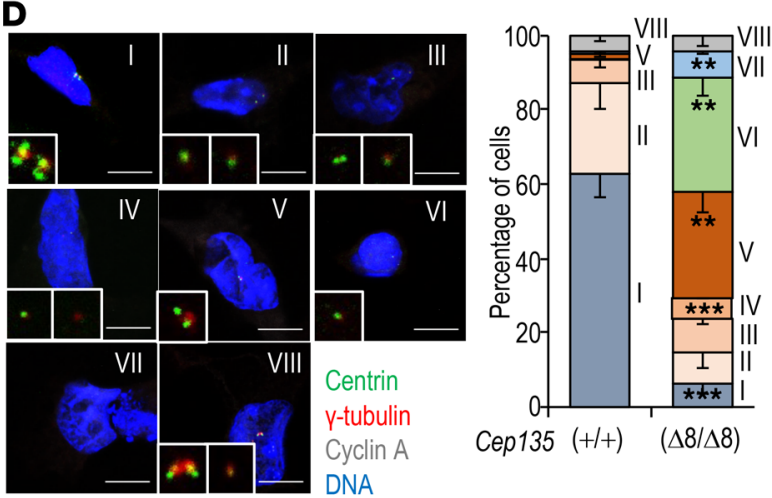

E
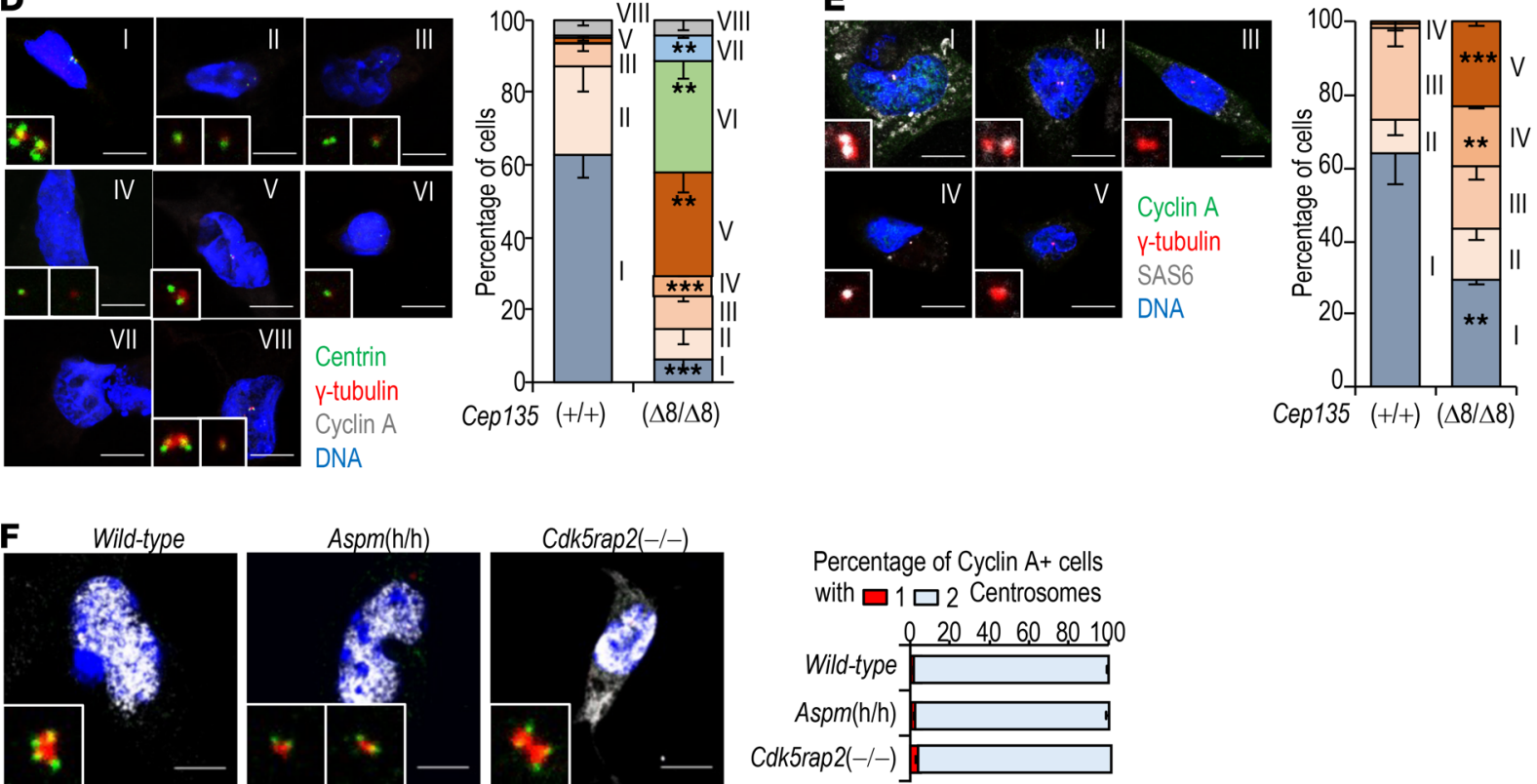

$\operatorname{Aspm}(\mathrm{h} / \mathrm{h})$
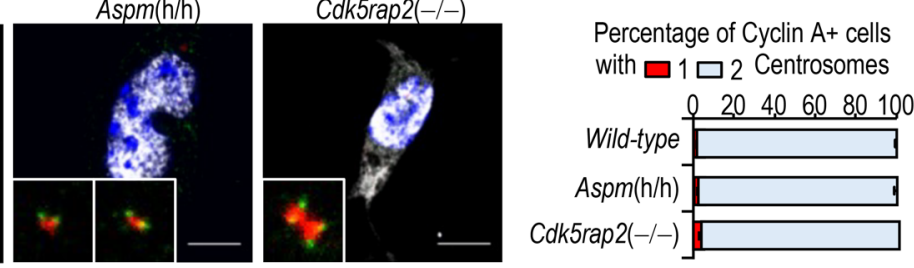

Percentage of Cyclin A+ cells

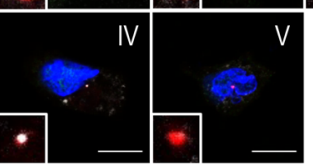

Cyclin A y-tubulin SAS6 DNA

C

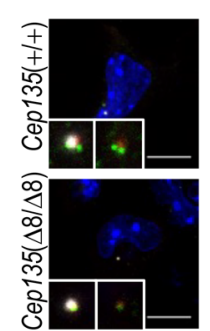

Centrin / y-tubulin /

80 ODF2 / DNA

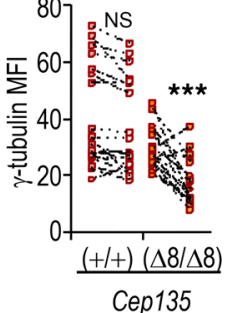

y-tubulin / Centrin / Cyclin A / DNA

Figure 2. Centrosome dynamics defects in Cep135-deficient fibroblasts. (A) Confocal imaging of E14.5 Cep135-mutant and control MEFs stained with the indicated markers. The histogram shows the percentage of cells with 1, 2, or more than $2 \gamma$-tubulin spots. (B) Immunostaining of MEFs with the indicated markers. The bottom histograms show the percentage of cyclin A+ cells with 1, 2, or more than $2 \gamma$-tubulin spots in the different phases of the cell cycle, as determined by PCNA and PH3 staining. (C) Immunostaining with antibodies against centrin (green), $\gamma$-tubulin (red), and ODF2 (cenexin, a marker of the mother centrosome; gray), and quantification of $\gamma$-tubulin mean fluorescence intensity (MFI). Slashed lines link 2 centrosomes from the same cell; higher slope of the line indicates higher centrosomal asymmetry. (D) Confocal imaging of E14.5 Cep135( $\Delta 8 / \Delta 8)$ MEFs. All cells depicted are cyclin A+. Group I: $2 \gamma$-tubulin spots; 2 centrin doublets. Group II: $2 \gamma$-tubulin spots; 2 centrin singlets. Group III: $2 \gamma$-tubulin spots; 1 centrin doublet + 1 centrin singlet. Group IV: $2 \gamma$-tubulin spots; 1 centrin doublet + 1 centrin singlet. Group V: $1 \gamma$-tubulin spot; 1 centrin doublet. Group VI: $1 \gamma$-tubulin spot; 1 centrin singlet. Group VII: acentrosomal. Group VIII: >2 $\gamma$-tubulin spots. (E) As in D. Group I: $2 \gamma$-tubulin spots; 2 Sas6 singlets. Group II: $2 \gamma$-tubulin spots; 1 Sas6 singlet + no Sas6. Group III: $2 \gamma$-tubulin spots; no Sas6 - no Sas6. Group IV: $1 \gamma$-tubulin spot; 1 Sas6 singlet. Group V: $1 \gamma$-tubulin spot; no Sas6. (F) Confocal imaging of E14.5 MEFs and percentage of cyclin A+ cells with 1 or $2 \gamma$-tubulin spots. Scale bars: $10 \mu \mathrm{m}$ (A-F). Data are mean \pm SEM from 3 cultures from 3 E14.5 embryos; $n>150$ cells/condition; ${ }^{* *} P<0.01 ;{ }^{* *} P<0.001 ; 1$-way ANOVA with Tukey's multiple comparisons (A, B, and F) and Student's $t$ test (C-E).

apical progenitor (AP) and the TBR2 ${ }^{+}$intermediate progenitor (IP) cell populations within the developing neocortex (Figure 3C and Supplemental Figure 4, A-D). No significant differences were observed in the orientation of the mitotic spindle in dividing cells of the AP layer in Cep135-deficient embryos (Supplemental Figure 4E). However, while most mitoses were bipolar in $C e p 135(+/+)$ embryos, $\operatorname{Cep} 135(\Delta 8 / \Delta 8)$ 
embryonic sections revealed frequent mitotic defects, including monopolar or acentrosomal mitotic spindles, in addition to apoptotic figures (Figure 3D and Supplemental Figure 4F). Maturation of centrosomes was impaired as detected by overall reduced $\gamma$-tubulin staining in APs of Cep135-deficient embryos (Figure 3, E and F). Cep 135( $\Delta 8 / \Delta 8)$ APs also presented asymmetric centrosomes with 1 of the 2 centrosomes in the poles with dim or almost absent $\gamma$-tubulin signal and asymmetric immunostaining of the distal appendage mother centriole protein ODF2 (Figure 3E). Transmission electron microscopy (TEM) analysis of centrioles in APs showed aberrant axial structure in $C e p 135(\Delta 8 / \Delta 8)$ embryos, with lack or abnormal number of microtubule triplets, reduced centriolar diameter in some centriolar structures, and lack of a clear 9-fold symmetric conformation (Figure 3G and Supplemental Figure 4G). These results suggest that alteration of CEP135 protein levels lead to defective centriolar duplication and dynamics in NPs, accompanied by MCPH.

A TP53 response in Cep135-deficient NPs. To further gain insight into the molecular pathways deregulated during cortical development in Cep135-mutant embryos, we subjected developing cortices from E11.5 and E14.5 Cep135( $\Delta 8 / \Delta 8)$ and control Cep135(+/+) embryos to RNA sequencing (RNAseq) analysis. In addition, E14.5 cortices were disaggregated and cultured to form incipient neurospheres, and RNA from these in vitro cultures was analyzed after 12 hours and 24 hours (Figure 4A). Combined analysis of transcriptomic profiles from these samples segregated 3 main groups corresponding to E11.5 cortices, E14.5 cortices, and cultured cells (12- and 24-hour time points; Figure 4B). Differential expression between Cep135-null and control samples in each group (Supplemental Figure 5A) suggested a defect in neural differentiation and function in Cep135-deficient samples both in vivo at E11.5 (Figure 4C, Supplemental Figure 5B, and Supplemental Table 1) and E14.5 (Supplemental Figure 5C and Supplemental Table 2), as well as in the neurosphere formation assay (Figure 4D, Supplemental Figure 5C, and Supplemental Tables 3 and 4). Interestingly, the TP53 pathway was deregulated in these samples, with significant upregulation of multiple TP53 target genes and a significant enrichment of deregulated transcripts with TP53 binding sites in their promoters (Figure 4, C and D; Supplemental Figure 5C; and Supplemental Tables 5-8). The TP53 target and cell cycle inhibitor Cdkn1a (p21 ${ }^{\text {Cip1 }}$ ) was among the most upregulated genes in these samples (Supplemental Table 9). Immunofluorescence studies in neurosphere cultures detected a significant presence of $\mathrm{TP}^{+} 3^{+}$cells in CEP135-deficient progenitors, as well as neurospheres cultured from CDK5RAP2-null progenitors (Figure 4E). This signal was accompanied by apoptotic cell death in neurosphere cells, as detected by active caspase 3 (Figure $4 \mathrm{~F}$ ).

TP53 was also significantly induced in several tissues in E11.5 Cep135( $\Delta 8 / \Delta 8)$ embryos (Figure 5A). This response was accompanied by a significant number of apoptotic cells (as detected by active caspase 3 ) in the developing brain and the rest of the body, especially dorsal telencephalon cortical cells and hematopoietic progenitors in the embryonic liver. At E14.5, apoptotic cells were observed in the developing neocortex but were almost absent in the rest of the embryonic tissues (Figure 5B). Three-dimensional immunostaining for cleaved caspase 3 in whole embryos suggested that apoptosis was especially evident in the developing neocortex at this stage (Figure 5C). Additional immunofluorescence studies for SOX2 and TUJ1 to detect APs and neuroblasts, respectively, showed apoptotic cell death in both progenitor and neuroblast lineages in the E14.5 developing neocortex (Figure 5D). E11.5 and E14.5 Cep135( $\Delta 8 / \Delta 8)$ embryos also displayed a reduced number of proliferating (as defined by $\mathrm{PH}^{+}$mitoses) cells in several tissues (Supplemental Figure 6, A and B), suggesting that both early apoptosis and defective proliferation may contribute to reduced body size in these mutant Cep135-deficient embryos. No evidence of apoptotic cells was observed in Cep135-mutant embryos at E17.5 (Supplemental Figure 6C), suggesting clearance of apoptotic cells and adaptation to the lack of CEP135 during the later stages of development. Both TP53 and active caspase 3 were also present in Cdk5rap2-deficient embryos, whereas these signals were only eventually seen in Aspm-deficient embryos (Figure 5E and Supplemental Figure 6, D and E).

TP53 protects Cep135-mutant brains from structural aberrations during development. We next tested the functional relevance of TP53 in the phenotype of Cep135-deficient embryos by interbreeding Cep135(+/ $\Delta 8)$ with TP53-deficient mice. Lack of TP53 (Supplemental Figure 7A) prevented apoptosis in E11.5 and E14.5 Cep135( $\Delta 8 / \Delta 8$ ); Trp53(-/-) double mutant embryos (Figure 6A and Supplemental Figure 7A). Intriguingly, TP53 loss did not rescue the reduced thickness of the neocortex in Cep135-null embryos (Supplemental Figure 7A). In fact, whereas we did not observe increased lethality of E11.5 Cep135( $\Delta 8 / \Delta 8)$; Trp53(-/-) double mutant embryos, we only recovered 1 double mutant embryo alive out of 39 E14.5 embryos derived from 4 matings between Cep135(+/ $\Delta 8)$; Trp53(-/-) males and Cep135(+/ $\Delta 8)$; Trp53(+/-) females. Two additional double mutant dead embryos were reabsorbed (Supplemental Figure 7B), suggesting premature embryonic 

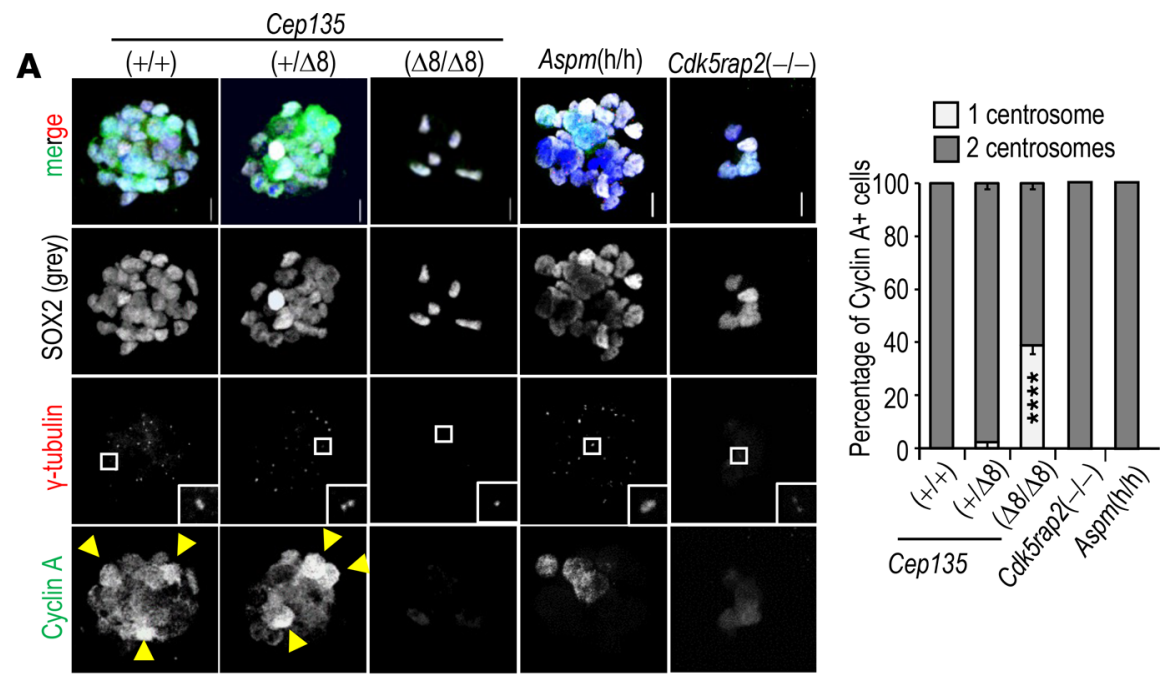

B

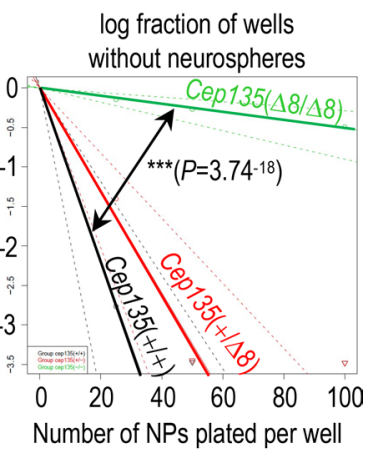

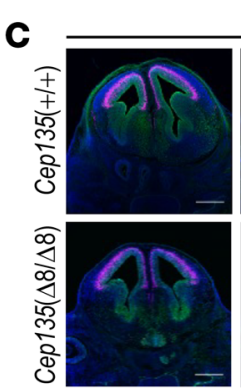

Rostral

SOX2 / TBR2 / DNA
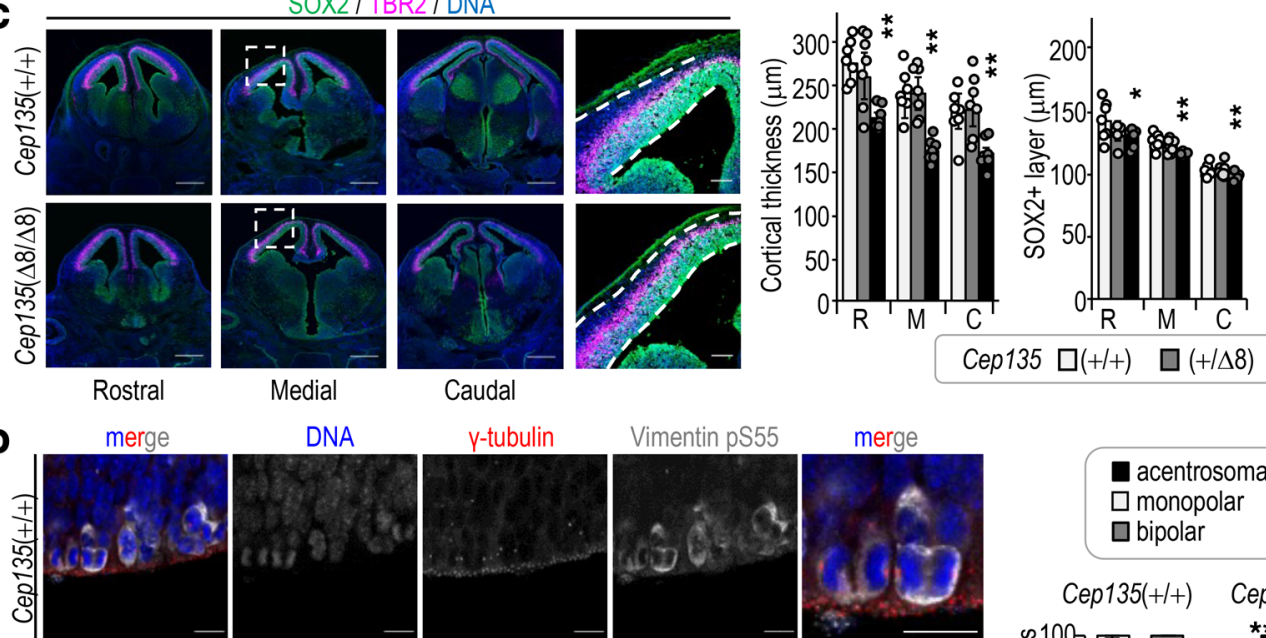

$200 \mid 8^{*} 0^{*}+*$

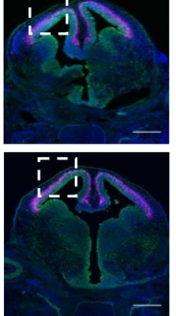

DNA

y-tubul
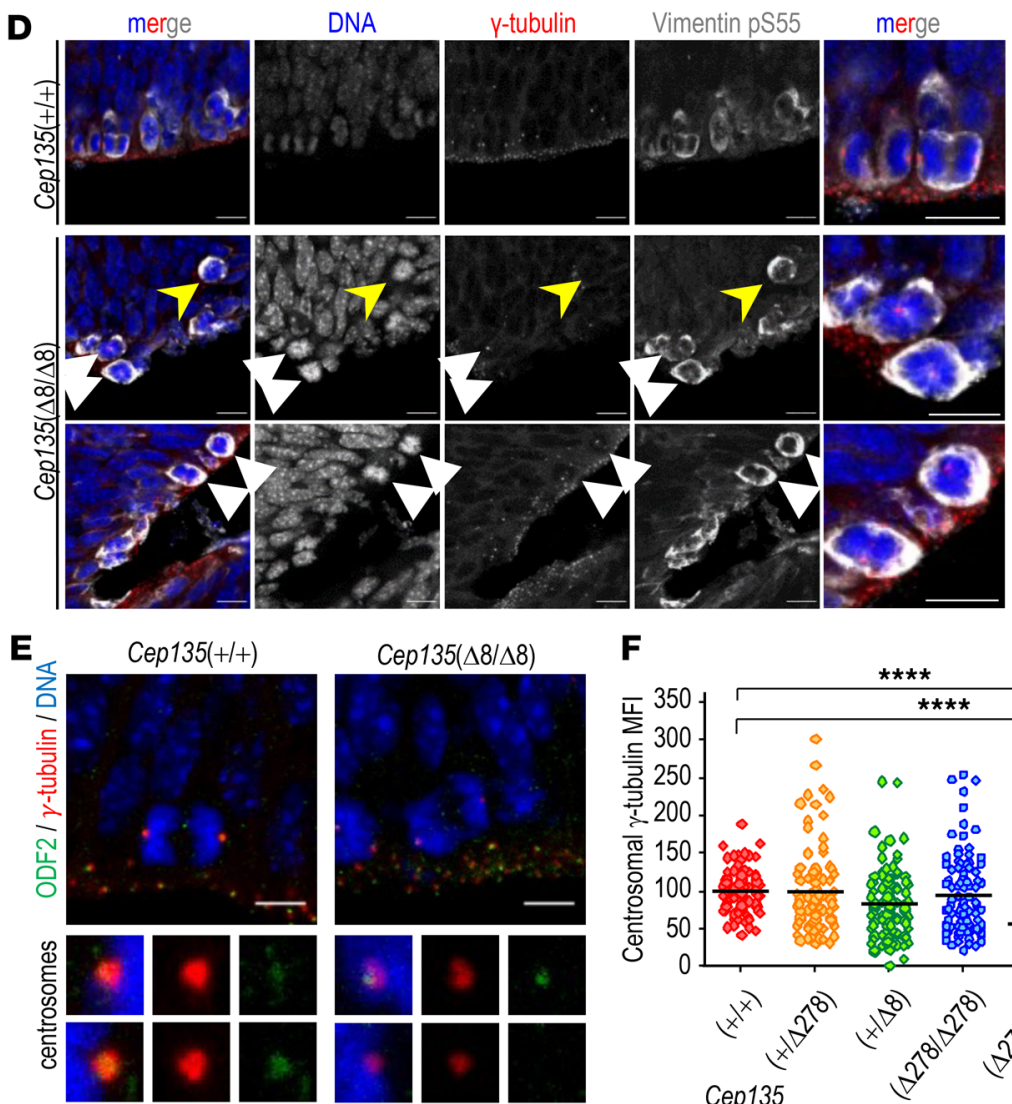

F
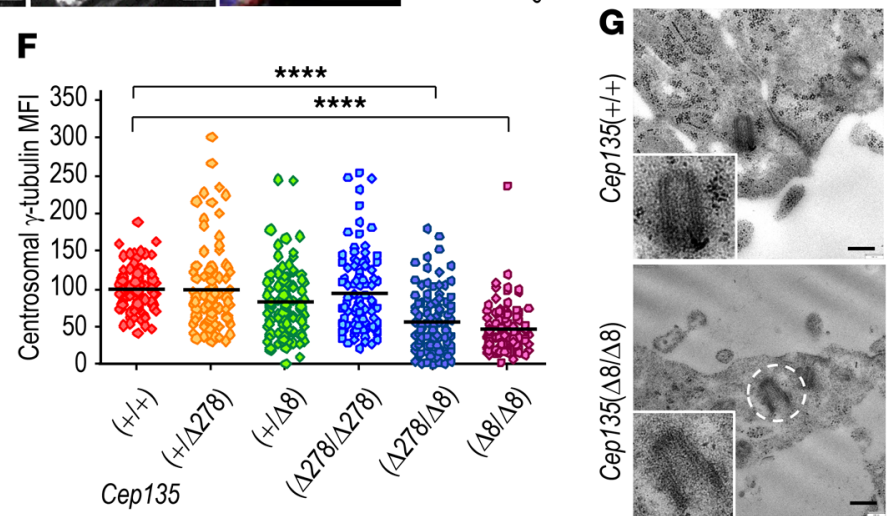

Figure 3. Self-renewal ability and centrosome dynamics in Cep135-mutant neural progenitors. (A) Confocal imaging of neurospheres derived from E14.5 embryonic cortices stained with the indicated antibodies, and percentage of cyclin A+cells with 1 or 2 centrosomes. (B) Self-renewal ability of neural progenitors as determined by limiting dilution assays. (C) Confocal imaging of cryosections of the rostral, medial, and caudal aspects of E14.5 embryonic 
brains with the indicated markers. Scale bar: $1 \mathrm{~mm}$ (left) and $100 \mu \mathrm{m}$ (insets). Quantification of the thickness (left), SOX2 ${ }^{+}$cells (middle), and TBR2 ${ }^{+}$cells (right) in rostral (R), medial (M), and caudal (C) regions. (D) Immunofluorescence with the indicated antibodies in developing E14.5 neocortices. Control samples show typical bipolar spindles, whereas monopolar spindles (white arrowheads in middle panels), acentrosomal spindles (yellow arrowheads), and asymmetric centrosomes (arrowheads in bottom panels) are observed in Cep135-mutant samples. Scale bars: $25 \mu \mathrm{m}$. The histogram shows the quantification of polarity in mitotic spindles (monopolar, bipolar, or acentrosomal) in ventricular or abventricular mitoses of E14.5 neocortices. (E) Immunostaining with the indicated antibodies of anaphases in the ventricular surface of E14.5 Cep135-mutant and control embryos. Higher-magnification images of mitotic centrosomes are displayed in the bottom panels. Scale bar: $10 \mu \mathrm{m}$. (F) Mean fluorescence intensity (MFI; arbitrary units) of $\gamma$-tubulin at the centrosome from NPs of the indicated genotypes. ${ }^{* * *} P<0.0001$ (unpaired $t$ test with Welsh correction). (G) Transmission electron micrographs showing representative pictures of centrioles contained in the first layer of APs of the ventricular surface. Scale bars: $200 \mathrm{~nm}$. Scale bars: $10 \mu \mathrm{m}$ (A, D, and E). Arrowheads indicate Cyclin A+ cells (A), TP53+ cells (B), or apoptotic cells (C). In A-D, data are mean \pm SEM. ${ }^{* *} P<0.01 ;{ }^{* *} P<0.001 ;{ }^{* * *} P<0.0001 ; 1$-way ANOVA with Tukey's multiple-comparison test (A, C, and $\mathbf{F}), \chi^{2}(\mathbf{B})$, Student's $t$ test (D).

death and embryonic reabsorption at midgestation. Ablation of Trp53 similarly prevented embryonic development of Cdk5rap2-deficient embryos, leading to earlier lethality ( E11.5; Supplemental Figure 7C).

The only E14.5 Cep135( $\Delta 8 / \Delta 8)$; Trp53(-/-) double mutant embryo presented profuse MCPH with severe cortical malformations, including abundant aberrant mitotic figures (Figure 6B), as well as perturbed laminar layering of NPs and abnormal neocortical architecture organization typical of subcortical heterotopias (Figure 6C). These abnormalities were accompanied by aberrant mitotic figures in SOX2 ${ }^{+}$progenitors (Figure 6D), as well as monopolar mitosis typical of centrosomal separation defects (Figure 6E). Finally, immunostaining of Cep135-mutant neocortices with the mitotic marker phospho-vimentin S55 and the proliferation marker Ki67 showed an increased number of mitotic figures in the ventricular zone (VZ) of Cep135( $\Delta 8 / \Delta 8)$; Trp53(+/+) and Cep135( $\Delta 8 / \Delta 8)$; Trp53(+/-) double mutant embryos, suggesting that centrosomal defects may be causative of impaired NPC division dynamics during neurodevelopment in these mutant brains (Figure 6F). Additionally, careful examination of the mitotic figures enriched in the Cep135( $\Delta 8 / \Delta 8)$; Trp53(+/-) brains revealed an increased frequency of acentrosomal mitoses compared with Cep135( $\Delta 8 / \Delta 8)$; Trp53(+/+) brains (Figure $6 \mathrm{~F})$, suggesting a role for TP53 in the clearance of these aberrant cells induced by $C$ ep 135 loss during brain development midgestation.

Due to the lethality of mutant mice with homozygous mutations in Trp53 and MCPH genes, we also tested the effect of partial ablation of Trp53 in a MCPH background. Elimination of 1 copy of Trp53 did not change the ratio of apoptotic cells (data not shown), nor the overall size of the brain in Aspm-, Cdk5rap2-, or Cep135-null newborns (Supplemental Figure 7D). However, Cep135( $\Delta 8 / \Delta 8)$; Trp53(+/-) mutant embryos presented cortical malformations (Figure 7A) together with severe cortical dysplasia and subcortical heterotopias within the neocortex (Figure 7B), suggesting that reduced levels of TP53 established a permissive threshold for Cep135-deficient cells to form these abnormal clumps of cells. Heterotopias were characterized by the presence of TBR1 and CTIP2 (postmitotic neuroblasts) and TBR2 (IP) positive cells (Figure 7C), a phenotype typically attributed to defects in radial migration of cortical projection neurons in models of autism or related heterotopias (24), as well as the presence of cells with abnormal nuclear morphologies, multilobulated nuclei, or abnormally large nuclei, suggestive of previous aberrations during chromosome segregation (Figure 7D). Interestingly, these heterotopias were characteristic of Cep135( $\Delta 8 / \Delta 8)$; Trp53(+/-) mutant embryos and were not observed in double mutants in Trp53 and other MCPH genes (Figure 7E). Together, these data suggest that CEP135 loss promotes defective centrosome duplication in NPs, leading to TP53-dependent cell death and $\mathrm{MCPH}$, as well as the accumulation of abnormal heterotopias in the presence of reduced TP53 levels.

\section{Discussion}

Despite the identification of more than $20 \mathrm{MCPH}$ genes causative of primary $\mathrm{MCPH}$, the cellular basis underlying the neurodevelopmental abnormalities present in patients with most of these specific mutations remains elusive. Interestingly, many $M C P H$ gene products are known to be located at the centrosome or spindle poles, pointing to centrosome dysfunction as one of the main causes of $\mathrm{MCPH}(4,25)$. The altered mitotic behavior commonly observed in MCPH usually comprises excessive asymmetric cell division of NPs during brain development, impaired neural differentiation, or cell death of progenitors, which accounts for a reduced pool of progenitors during development, reduced neuronal output, and as a result, MCPH $(25,26)$.

Biallelic single-bp deletions of the human CEP135 gene have been reported in MCPH patients with significant $\mathrm{MCPH}$ concomitant with severe intellectual disability and communication deficits (17, 27). Previous reports included CEP135 in a group of centriolar proteins, including Sas 4 and Sas6, thought to be 
A

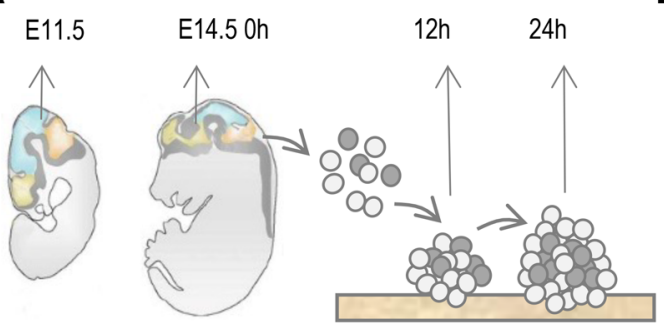

C

\begin{tabular}{lc}
\hline Deregulated pathways & \\
\hline Cluster of Pathways & Enrichment Score \\
\hline TP53, microRNAs and cell cycle & 1.6991 \\
Differentiation \& neurogenesis & 1.6960 \\
Transcriptional misregulation & 0.7979 \\
Cell adhesion & 0.3413 \\
\hline
\end{tabular}

B

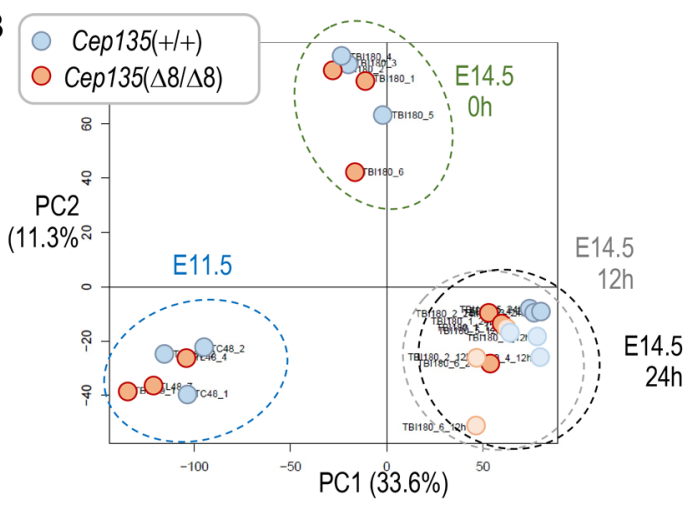

\begin{tabular}{ll}
\multicolumn{2}{c}{ Enrichment in TF targets } \\
\hline Enriched term & $p$ value \\
\hline TP53 Chea & 0.002 \\
KLF4 Chea & 0.006 \\
SOX2 Chea & 0.009 \\
SUZ12 Encode & 0.014 \\
NANOG Chea & 0.018 \\
\hline
\end{tabular}

E14.5-24h

\begin{tabular}{lc}
\hline Deregulated pathways & \\
\hline Cluster of Pathways & Enrichment Score \\
\hline Nervous system dev. \& differ. & 3.5012 \\
Cell junction \& Synapse & 2.8047 \\
Calcium signaling & 2.4199 \\
WNT, Hippo \& cancer pathways & 1.9724 \\
Regulation of transcription & 1.7160 \\
\hline
\end{tabular}

\begin{tabular}{lc}
\multicolumn{2}{c}{ Enrichment in TF targets } \\
\hline Enriched term & Adj.p value \\
\hline Suz12 Chea & 0.000 \\
TP53 Chea & 0.001 \\
TCF3 Chea & 0.003 \\
SUZ12 Encode & 0.006 \\
NFE2L2 Chea & 0.030 \\
\hline
\end{tabular}

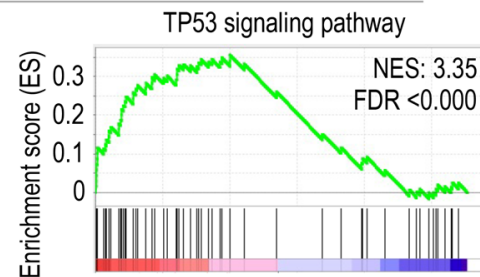

Rank in ordered dataset

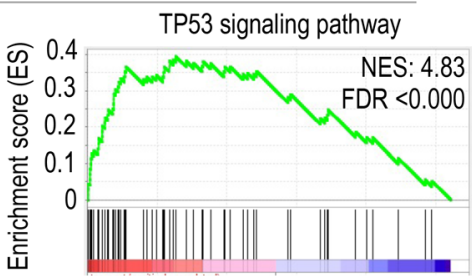

Rank in ordered dataset
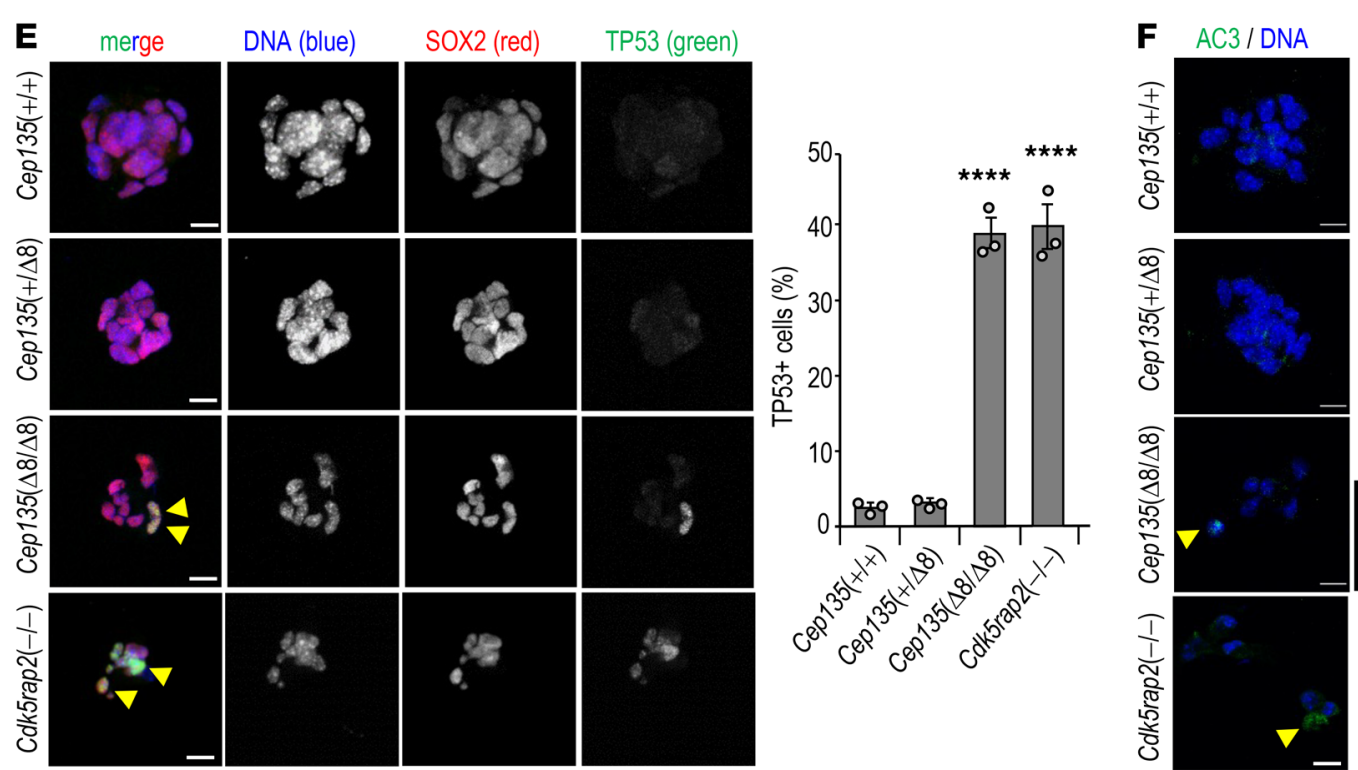

Figure 4. TP53-mediated response to lack of Cep135 in developing brains and cultured neurospheres. (A) Schematic representation of the Cep135(+/+) and Cep135( $\Delta 8 / \Delta 8)$ samples selected for RNA sequencing analysis, including E11.5 and E14.5 brains, as well as neurospheres from E14.5 brains cultured for 12 and 24 hours. (B) Principal component analysis of the transcriptomic profiles from the indicated samples. Values in PC labels show the percentage of explained variance for principal component 1 (PC1) and PC2. (C) Major pathways deregulated and enrichment in transcription factor (TF) targets in E11.5 samples. The enrichment in transcripts involved in the TP53 pathway is shown to the right. (D) Major pathways deregulated and enrichment in TF targets in E14.5 samples cultured during 24 hours to form neurospheres. The enrichment in transcripts involved in the TP53 pathway is shown to the right. See Supplemental Tables 1-9 for additional details. (E) Immunostaining of primary neurospheres from E14.5 embryos with SOX2 and TP53 antibodies. The percentage of TP53+ cells is shown to the right. (F) Confocal imaging of primary neurospheres stained with antibodies against active caspase 3 (AC3, green). DAPI (DNA) is shown in blue. Scale bars: $10 \mu \mathrm{m}$ (E and $\mathbf{F})$. Yellow arrowheads indicate TP53 $(\mathbf{E})$ and CC $3^{+}$cells $(\mathbf{F})$. In $\mathbf{E}$, data are mean \pm SEM. ${ }^{* * *} P<0.0001$ (1-way ANOVA with Tukey's multiple-comparison test). 
A $\quad \mathrm{E} 11.5$
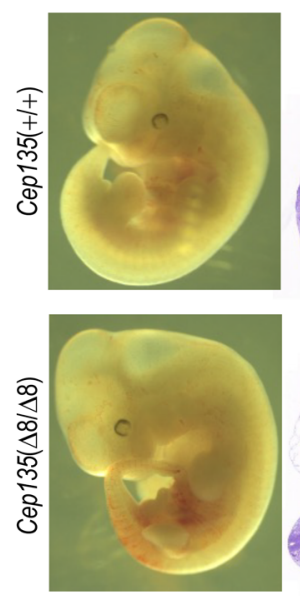
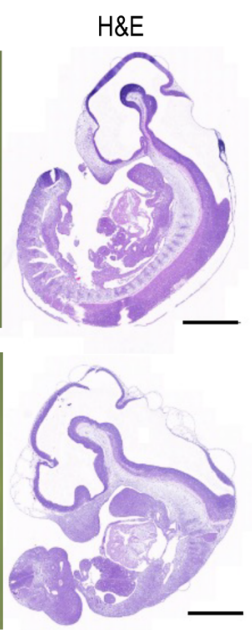
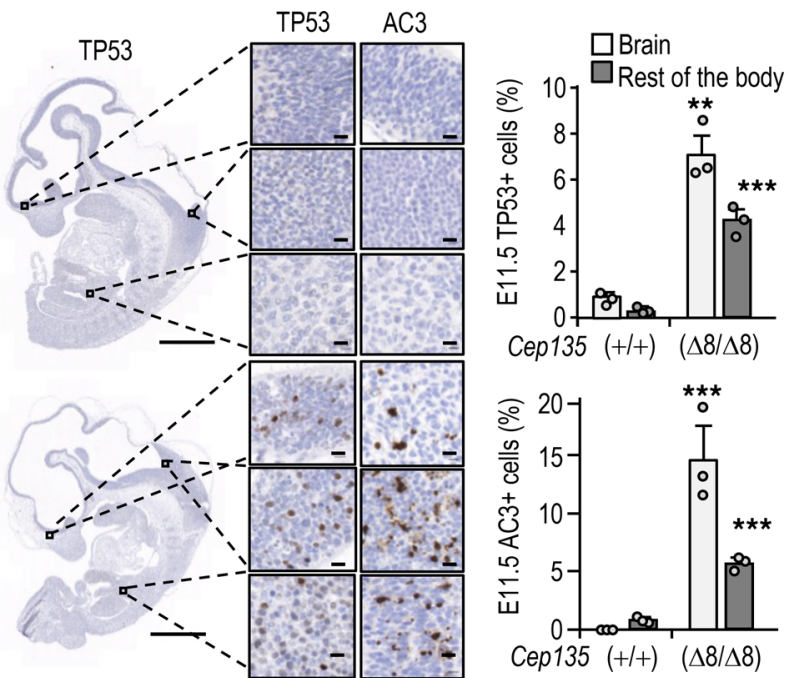

Cep135 $(+/+)(\Delta 8 / \Delta 8)$

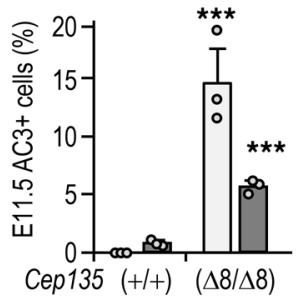

B $\quad$ E14.5
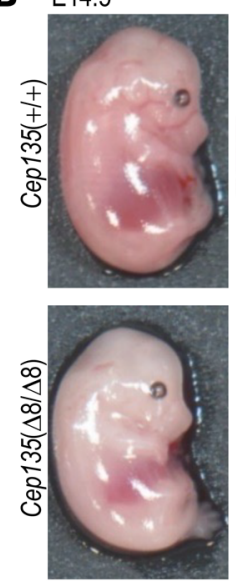

C
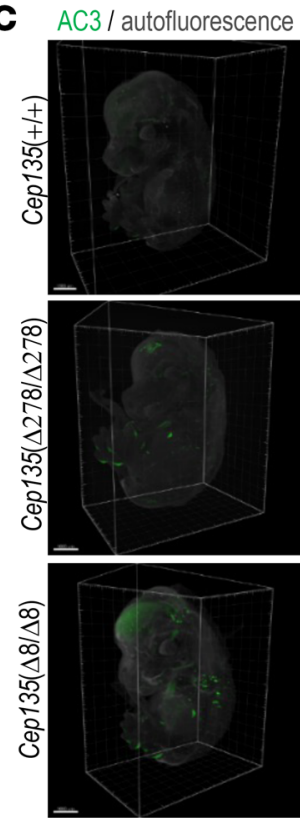
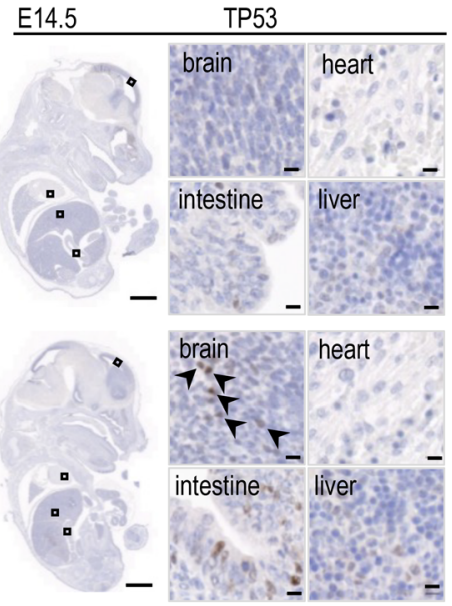
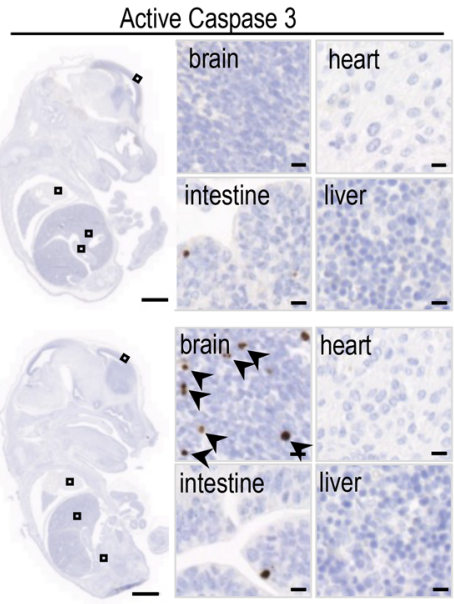

Dorsal neocortex
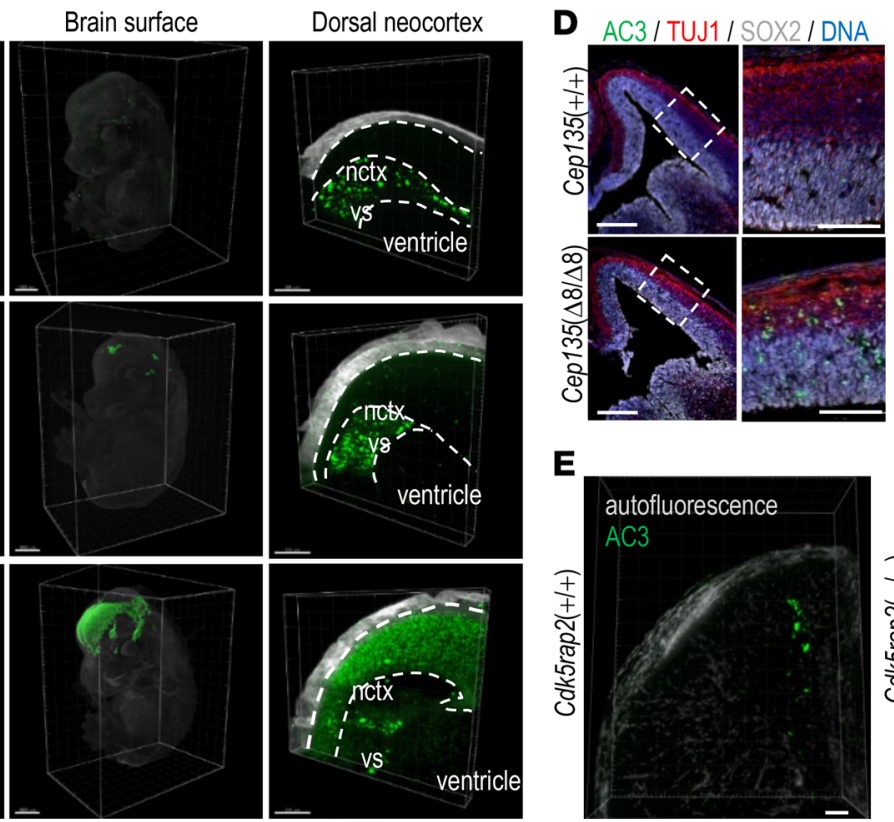
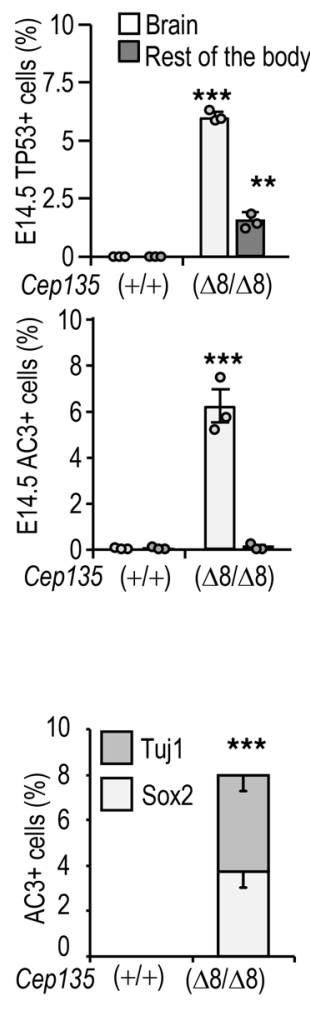

Figure 5. Cep135 deficiency induces TP53-dependent apoptosis in neural progenitors. (A) Bright-field macroscopic (left) and H\&E staining (middle) images of E11.5 Cep135(+/+) and Cep135( $\Delta 8 / \Delta 8)$ mouse embryos. IHC staining for TP53 and active caspase 3 (AC3) in the same samples including insets at higher magnification (TP53, left; AC3, right) from the specific areas (forebrain [upper], medullary hindbrain [middle], and liver [bottom]). The quantification of $\mathrm{TP}^{2} 3^{+}$and $\mathrm{AC}^{+}$cells in brain and rest of the body of Cep135-mutant and control embryos is shown in the right 
histograms. Scale bars: $1 \mathrm{~mm}$ (whole embryo sections) and $10 \mu \mathrm{m}$ (insets). (B) Bright-field macroscopic images (left), IHC staining (middle), and quantification (right) of TP53+ and $\mathrm{AC3}^{+}$cells in brain and rest of the body of E14.5 Cep135-mutant and control embryos. Scale bars: 1 mm (whole embryo sections), $100 \mu \mathrm{m}$ (lower microscopic insets). Representative positive cells are indicated by arrows. (C) Whole-mount 3D immunofluorescence of E14.5 Cep135(+/+), Cep135( $\Delta 278 / \Delta 278)$, and Cep135( $\Delta 8 / \Delta 8)$ mouse embryos stained for AC3 (green). Gray color depicts tissue autofluorescence in the 488 channel. Scale bars: $1 \mathrm{~mm}$ (whole embryos) and $200 \mu \mathrm{m}$ (lower microscopic insets). Nctx, neocortex; vs, ventricular surface. Note that green, unspecific positive signal in the vs of Cep135(+/+) samples corresponds to secondary antibody aggregates. (D) Immunostaining for AC3, SOX2, and TUJ1 in 14.5 neocortex from Cep135-null and control mice. Scale bars: $250 \mu \mathrm{m}$ (left) and $100 \mu \mathrm{m}$ (right). The bottom histogram shows the quantification of $\mathrm{AC}^{+}$cells in these samples. (E) Whole-mount 3D immunofluorescence of E14.5 Cdk5rap2(-/-) mouse embryos stained for AC3 (green). Scale bars: $200 \mu \mathrm{m}$. In A, B, and D, data are mean SEM from 3 different embryos; ${ }^{* *} P<0.01$; ${ }^{* * *} P<0.001$ by Student's $t$ test.

essential for centriole assembly $(8,9,25)$. Several studies have delineated a WDR2/ASPM/SAS4/SAS6 pathway in which these $\mathrm{MCPH}$-associated proteins recruit each other sequentially to the centrosome, thereby enabling centriole duplication to occur $(25,28,29)$. CEP135 interacts with SAS6, as well as tubulin, participating in the interaction between the cartwheel and microtubule triplets (30).

Germline ablation of Sas 4 in the mouse results in severe defects in centriole biogenesis and duplication, leading to embryonic death as soon as E8.5 (31). Conditional genetic depletion of these core structural proteins in later stages of embryonic development alter brain neurodevelopment by promoting AP detachment from the ventricular surface, which results in spindle orientation randomization, ectopic proliferation of APs, and progressive loss of centrioles concomitant with TP53 activation and apoptotic death (32). Sas4-null cells lack centrosomes and primary cilia; however, acentriolar spindle poles are assembled, and these mutant cells do not display obvious defects in chromosome segregation (apart from slightly prolonged mitosis) or cell cycle profiles (31). Similarly, genetic ablation of SAS6 in human cells results in lack of centrioles (33). Whether similar requirements apply to mammalian CEP135, however, is less clear. Pioneering in vitro studies of Cep135 deficiency using homologous recombination in vertebrate cell lines reported no significant alterations of centriole structure, dynamics, or cell division, although a small decrease in centriole numbers and increase in the frequency of monopolar spindles was observed (15). In contrast to these reports, we observed that complete genetic depletion of Cep135 in vivo induces significant defects in centriole duplication, leading to frequent apoptotic cell death in several tissues by midgestation. These defects are more pronounced and lasted longer in NPs at later embryonic stages, ultimately leading to MCPH. As opposed to Sas4-KO mice, which die before midgestation $(31,32)$, Cep135-deficient mice progressed through embryonic development until birth, indicating that - unlike SAS4 - CEP135 is dispensable for early embryonic development. The abnormal cell division of NPs midgestation in the absence of CEP135 was accompanied by a TP53 transcriptional response and TP53-dependent apoptotic cell death. Ablation of the Trp53 gene prevented apoptotic cell death but did not rescue the developmental defects caused by Cep 135 ablation. This is in contrast to previous MCPH models in which the developmental delay and MCPH caused by mutations in Sas4 (31, 32), Kif20 (34), or Cep63 (35) were rescued after Trp53 ablation. The reasons for these differences are unclear but suggest that Cep135 ablation may alter additional TP53-independent pathways in the developing cortex.

Taken together, these data indicate that CEP135 depletion promotes centrosome duplication defects and mitotic aberrations, accompanied by TP53 activation and defective production of neurons during brain development. The resulting MCPH (25\% decrease of cortical area in Cep135-mutant newborns) can be considered as severe, taking into consideration the ratio between human and murine encephalization, similar to other genetic mouse models based on $M C P H$ gene mutations. The mechanism underlying Cep135-null phenotypes - i.e., defective centriole duplication - may, however, be relatively specific. Previous studies have shown that depletion of Aspm, which accounts for approximately $60 \%$ of human cases of $\mathrm{MCPH}$, in mice promotes mild $\mathrm{MCPH}$ and germline defects and affects spindle orientation and astral microtubule dynamics $(19,36,37)$. Cdk5rap2 loss also generates severe $\mathrm{MCPH}$ and dwarfism by affecting cell division, causing cell death of NPs during development (21).

Despite the frequency of centrosomal defects characteristic of the perturbed neural development observed in $\mathrm{MCPH}$ patients, it remains to be resolved why brain size, in particular, is so vulnerable to these defects $(4,25)$. Our observations in midgestation embryos suggest that the abnormal divisions and TP53 response to Cep135 loss are present in additional tissues, such as the embryonic liver. However, TP53 induction and apoptosis is only present in the developing cortex at later developmental stages. It, thus, appears that - compared with other organs - adaptation to centrosome defects is less efficient in the developing neural system, perhaps at least partially explaining the tight correlation between mutation in genes encoding centrosomal proteins and primary $\mathrm{MCPH}$. 
A

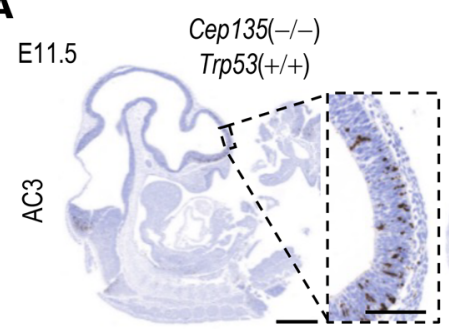

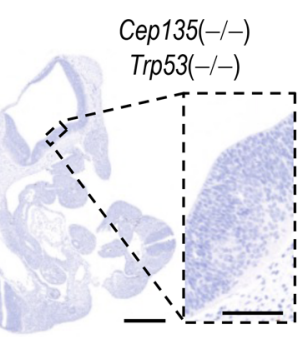

B

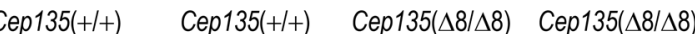
E14.5 Trp53(+H)

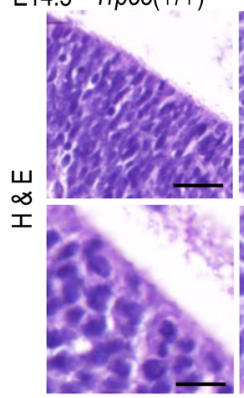

Cep135(+/+)

D

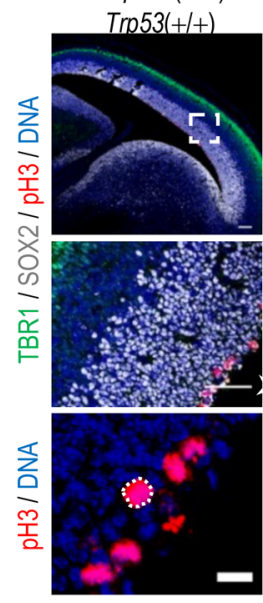

$\mathbf{F}$

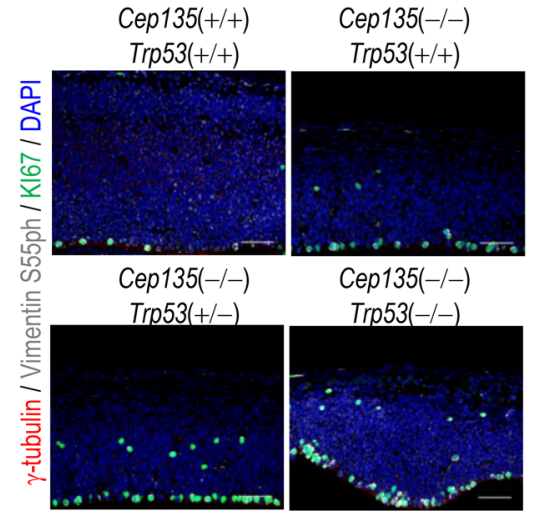
Trp53(-1-)

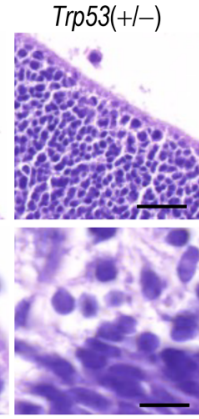

$\operatorname{Trp53}(-1-)$
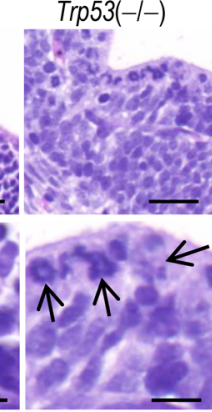

$\operatorname{Cep135}(\Delta 8 / \Delta 8) \quad \operatorname{Cep135}(\Delta 8 / \Delta 8)$
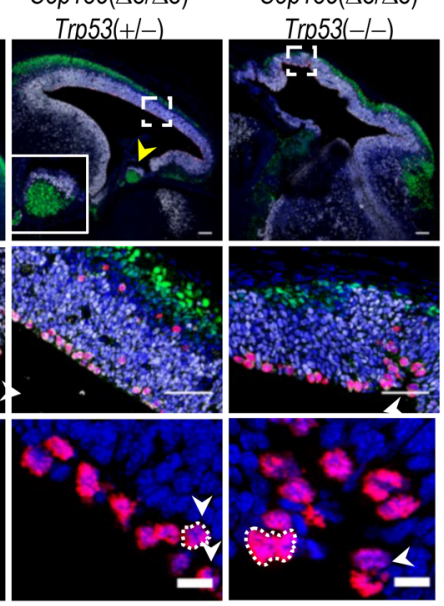

Cep135(+/+); Trp53(+/+

Cep135(-/-); Trp53(-/-)
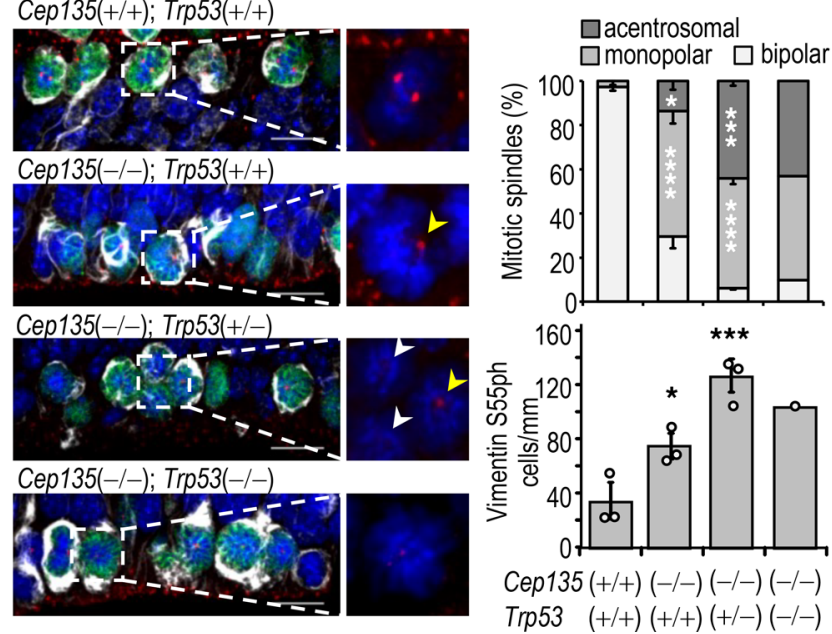

Figure 6. Genetic ablation of Trp53 in Cep135-K0 embryos. (A) IHC staining for active caspase 3 (AC3; brown) in the indicated models. Scale bars: 1 mm (whole embryos) and $100 \mu \mathrm{m}$ (insets). In the quantification, data are mean SEM (3 embryos). ${ }^{* *} P<0.01$; ${ }^{* * *} P<0.001$ by Student's $t$ test. (B) H\&E staining of the medial aspect of the neocortex of E14.5 embryos with the indicated genotypes. Scale bars: $20 \mu \mathrm{m}$ (upper left), $10 \mu \mathrm{m}$ (lower), and $100 \mu \mathrm{m}$ (right). Arrows indicate aberrant mitotic figures of apical progenitors. Scale: $10 \mu \mathrm{m}$. (C) Histological sections of E14.5 neocortices of the indicated genotypes showing cortical heterotopias and malformations (arrowheads). Scale bars: $100 \mu \mathrm{m}$ (upper) and $50 \mu \mathrm{m}$ (insets). (D) Confocal imaging of E14.5 neocortices 
stained with the indicated antibodies (upper), and higher magnification confocal images showing the neocortical area delimited by slashed boxes (middle) Yellow arrowhead indicates a subcortical heterotopia. Higher-magnification inset in the middle panel shows CTIP2+ heterotopias. Bottom panels show APs with aberrant mitotic figures (arrowheads). Scale bars: $100 \mu \mathrm{m}$ (upper), $50 \mu \mathrm{m}$ (middle), and $10 \mu \mathrm{m}$ (lower). (E) Confocal images of APs in the neocortices shown in $\mathbf{D}$ stained with the indicated antibodies. Scale bars: $10 \mu \mathrm{m}$. (F) Confocal micrographs of E14.5 neocortices stained with the indicated antibodies. The middle panels show higher-magnification images of the innermost APs with representative mitoses (insets) and monopolar spindles (yellow arrowheads), and acentromal cells (white arrowheads). Scale bars: $50 \mu \mathrm{m}$ (left), $10 \mu \mathrm{m}$ (middle), $10 \mu \mathrm{m}$ (right). Asterisks indicate comparison with Cep135(+/+); $\operatorname{Trp53}(+/+)$ controls. Data are mean SEM from 3 embryos (1 for Cep135; Trp53 homozygous mutants). ${ }^{*} P<0.01 ;{ }^{* *} P<0.001$; ${ }^{* * *} P<0.0001 ; 1-$ way ANOVA with Tukey's multiple-comparison test.

Although MCPH does not generally present with gross morphological defects in the neocortex, some cases with structural changes have been reported, including cortical malformations and subcortical heterotopia in patients with mutations in WDR62 and CENPJ (MCPH6) $(38,39)$. Interestingly, subcortical heterotopia has been recently reported in a CEP135 patient presenting with epilepsy (18). All these 3 proteins work together in centrosomal microtubule assembly. Interestingly, similar subcortical heterotopias group 1a have been associated with mutations in the genes encoding the katanin subunit KATNB1, also involved in microtubule severing at the centrosome, and the microtubule subunit tubulin $\beta$ (TUBB), suggesting a link between microtubule assembly and these brain malformations (39).

Cep135-deficient mutant embryos do not display heterotopias in the presence of a normal TP53 pathway. As these mutant mice die perinatally, we could not compare this phenotype with the heterotopias observed in the 18-year-old MCPH8 patient (18). However, heterotopias were observed in Cep135-mutant embryos with a defective TP53 pathway. Although the functional status of TP53 has not been analyzed in the MCPH8 patient, the CEP135 mutation was accompanied by other mutations in the same patient, including FAT4, a gene previously related to heterotopias. Thus, the causal role of CEP135 could not be completely established. Multifocal neocortical dysplasias are also common features of autism and underlying causes of epilepsy, which shows strong comorbidity with autism (40). Our data suggest that subcortical heterotopia may arise from centriolar duplication defects in Cep135-deficient cells, especially when TP53 is not efficient enough to eliminate aberrant cells during brain development, resulting in a combined phenotype of $\mathrm{MCPH}$ and heterotopia. It remains to be tested whether subcortical heterotopia in patients with CEP135 mutations may arise as a consequence of defective gatekeepers such as TP53 or cooperating mutations (e.g., FAT4; ref. 18) that may contribute to the development of this pathology.

\section{Methods}

Generation of mutant mice. Two different CRISPR sgRNAs were designed for generating null alleles as follows: Cep135_sg\#1:5'-AGTATATTAACATTCGGAAG-3'; Cep135_sg\#2:5'-CGGAAGAGGTTAGACCAGCT-3'; Aspm_sg\#1: 5'-TGGCGACAAAACGGGATTGA-3'; Aspm_sg\#2: 5'-GGACACGTAGGTCAGCAAAC-3'; Cdk5rap2_sg\#1 5'-GTCCTTCATGTTCCGGGCTC-3'; Cdk5rap2_sg\#2: 5'-TTCATGTTCCGGGCTCTGGT-3'. Ribonucleoprotein complexes were assembled by incubating Cas9 protein (LabOmics) (100 ng/mL) and $50 \mathrm{ng} / \mathrm{mL}$ of each sgRNA in microinjection buffer (10 mM Tris pH 7.4, 0.1 mM EDTA) for $15 \mathrm{~min}-$ utes at room temperature (RT). The ribonucleoprotein complexes were injected in the cytoplasm of zygotes obtained from matings of B6.CBA male and female mice at 0.5 days of gestation, using protocols previously described (41). Forty-five zygotes were injected for each model, from which 9, 21, and 18 pups were born for Cep135, Cdk5rap2, and Aspm models, respectively. Genotyping was performed by PCR and sanger sequencing. The genotyping oligonucleotides were as follows: Aspm (Fw: 5'-AGTCCCCACCCCATCCATTAG-3'; Rv: 5'-GTTGGCGTATACCCCCGAGT-3'), Cdk5rap2 (Fw: 5'-ACCACCAAGCAGGAAGTTGC-3'; Rv: 5'-AGTCATGTCAGAGTCCGGGG-3'), Cep135 (Fw: 5'-TTACTGTCACTAAGGGGTGCC-3'; Rv: $5^{\prime}$-CGTTACTCCCACGCAACTTC- $3^{\prime}$ ), and PCR conditions for the 3 genes indicated were $95^{\circ} \mathrm{C} 5$ minutes; $\left(95^{\circ} \mathrm{C}, 30\right.$ seconds; $60^{\circ} \mathrm{C}, 30 \mathrm{~s} ; 72^{\circ} \mathrm{C}, 60$ seconds) for 40 cycles; and $72^{\circ} \mathrm{C}$ for 10 minutes. Trp 53 -KO mice were obtained from the Jackson Laboratory (B6.129S2-Trp53tm1Tyj/J; stock no. 002101). Mice were maintained on a C57BL/6J genetic background. For histological examination, samples were fixed in a solution of $10 \%$ of buffered formalin (MilliporeSigma), embedded in paraffin, and cut into $2.5 \mu \mathrm{m}$ sections. The sections were then stained with H\&E or Cresyl violet (for Nissl staining).

Molecular imaging. Computerized axial tomography scanning (CT scan) was performed on anesthetized P30 mice [ $3 \%$ of Isoflurane (Isoba Vet)] using a PET-CT scan (GE Healthcare) with the following parameters: $200 \mathrm{~mA}, 35 \mathrm{kV}, 160 \mathrm{~m}, 16$ shots and 360 projections. MMWKS (GE Healthcare) and MicroView software were used to analyze the resulting images. 
A E14.5 Cep135( $\Delta 8 / \Delta 8) ;$ Trp53(+/-) TBR1 SOX2 pH3 DNA
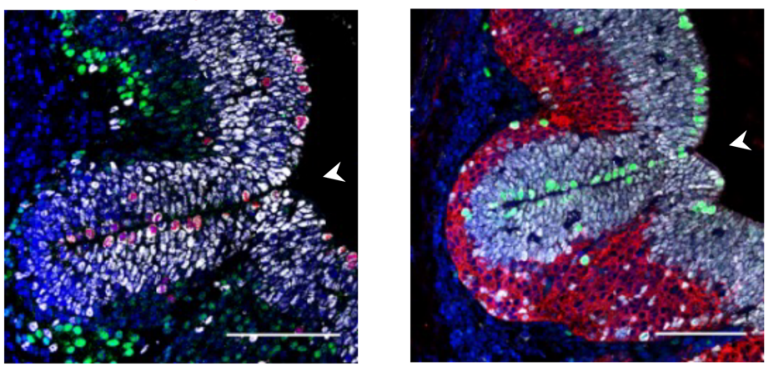

B

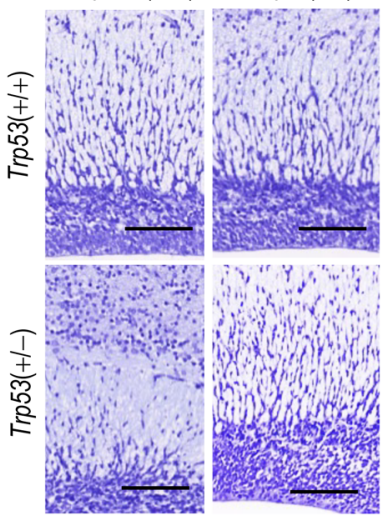

Cdk5rap2(-l-) Cep135( $\Delta 8 / \Delta 8)$

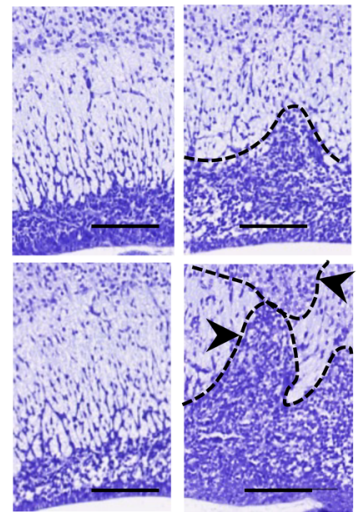

\section{c}

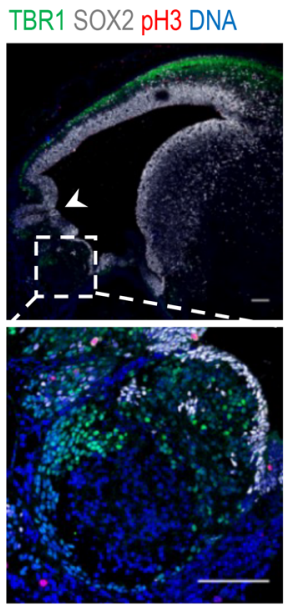

TBR2 SOX2 DNA

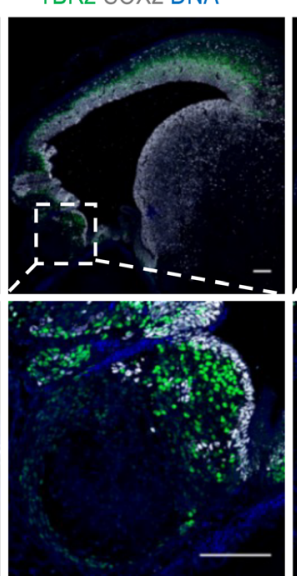

CTIP2 TBR1 DNA

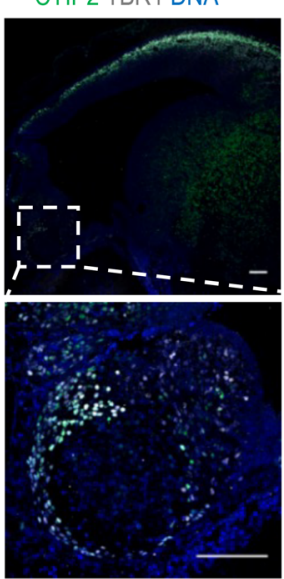

KI67 SOX2 TUJ1 DNA

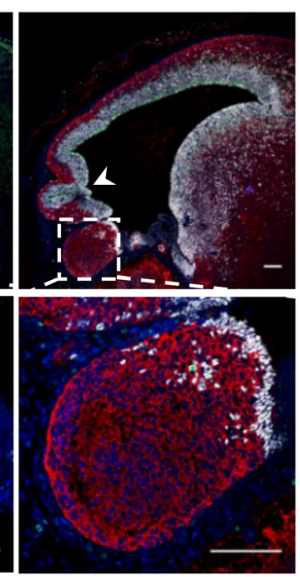

D TBR1 SOX2 pH3 DNA

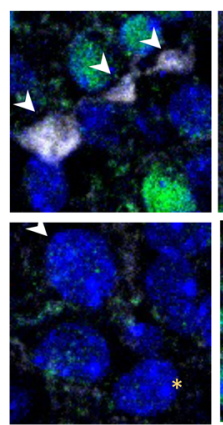

E14.5 Cep135( $\Delta 8 / \Delta 8) ; \operatorname{Trp53(+/-)~}$

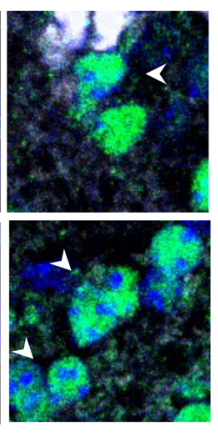

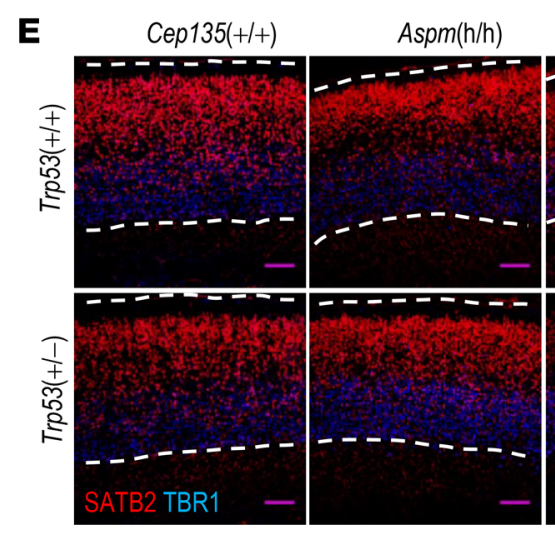
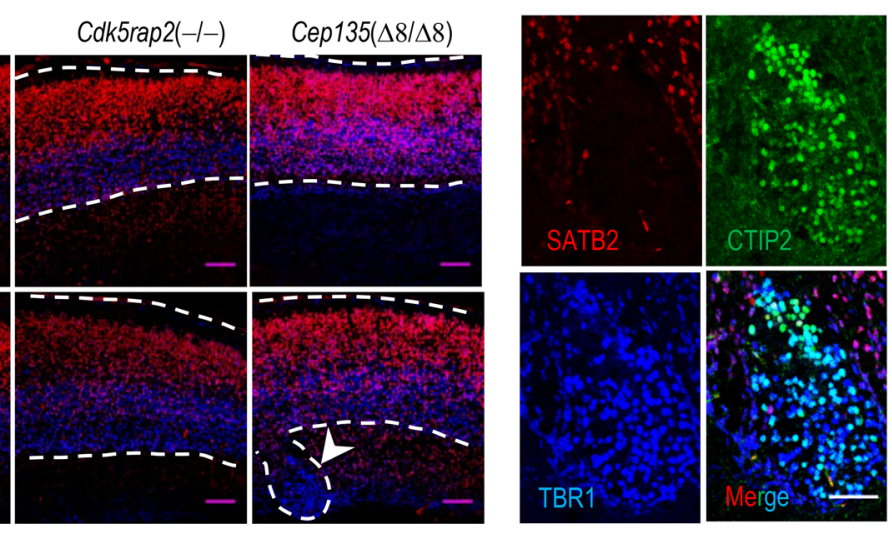

Cep135( $\Delta 8 / \Delta 8)$

$\operatorname{Trp53(+/-)}$

SATB2

CTIP2

TBR1

Figure 7. Partial depletion of Trp53 exacerbates the presence of cortical heterotopias in Cep135-mutant newborns but not in other MCPH models. (A) Confocal imaging of a E14.5 Cep135( $\Delta 8 / \Delta 8) ; T p 53(+/-)$ neocortex stained with the indicated antibodies. Arrowheads indicate regions of cortical malformations with aberrant folding. Scale bar: $100 \mu \mathrm{m}$. (B) Histological Nissl staining of PO cortices from newborns of the indicated genotypes, showing heterotopias in Cep135( $\Delta 8 / \Delta 8$ ); Trp53(+/-) double mutant newborns (arrowheads). Scale bar: $100 \mu \mathrm{m}$. (C) Immunostaining of E14.5 embryonic brain in seriated sagittal sections with the indicated antibodies. Arrowheads in upper confocal images depict regions of cortical malformations (also showed in A). Squared regions depict a cortical heterotopia, showed in higher magnification in lower panels. Scale bars: $100 \mu$ m. (D) Higher-magnification confocal images of specific cells from the previous panels showing aberrant and polylobulated nuclei (top arrowheads) and abnormally large nuclei (bottom arrowheads) cells in double mutant heterotopias. A normal nucleus (asterisk) is shown for comparison. Scale bar: $10 \mu \mathrm{m}$. (E) Immunostaining of PO neocortices of the indicated genotypes against SATB2 (layers II-V), CTIP2 (layer V), and TBR1 (layer VI). Note the presence of CTIP2+, TBR1 ${ }^{+}$, SATB2subcortical heterotopias. Scale bar: $100 \mu \mathrm{m}$ (left panels) and $50 \mu \mathrm{m}$ (higher-magnification panels to the right).

IHC. Tissues were fixed in 10\% buffered formalin (MilliporeSigma) and embedded in paraffin for routine histological analysis. IHC was performed on $2 \mu \mathrm{m}$ paraffin sections by using an automated protocol developed for the DISCOVERYXT-automated slide-staining system (Ventana Medical Systems Inc.). All steps were performed in this staining platform by using validated reagents, including deparaffinization, acidic antigen retrieval, 
and antibody incubation and detection. Primary antibodies used for this purpose are listed in Supplemental Table 10. Appropriate biotinylated secondary antibodies were used to detect the primary antibodies, followed by incubation with streptavidin-horseradish peroxidase and diaminobenzidine system. Full slides were digitalized with a Zeiss AxioScan Z1 and analyzed by using the ZEISS Zen 2.3 Imaging Software (Zeiss).

Immunofluorescence. Immunohistofluorescence was performed in mouse embryos or newborns tissues. Briefly, mouse embryos or newborns (P0) were decapitated, and brains were dissected out (in the case of P0 newborns). Embryonic heads or P0 brains were fixed in 4\% paraformaldehyde (electron microscopy sciences, 50-980-487) for 3 hours at RT and then overnight at $4^{\circ} \mathrm{C}$. Next, tissues were rinsed in PBS (MilliporeSigma) 3 times, immersed in 30\% sucrose (MilliporeSigma) for 48 hours at $4^{\circ} \mathrm{C}$, embedded in Tissue Tek OCT compound (Sakura), and cryosectioned onto SuperFrost Plus slides in a Leica cryostat in cryosections that were 12-14 $\mu \mathrm{m}$ thick. For immunostaining, cryosections were rehydrated and boiled in a microwave oven for epitope retrieval (if indicated) in sodium citrate buffer (10 $\mathrm{mM}$ [pH 6]) for 1 minute, maximum. Cryosections were equilibrated in PBS, permeabilized with Triton X-100 0.5\% for 5 minutes, washed, and incubated with blocking solution (4\% normal goat serum [Vector Laboratories, S-2000] in PBS-T [PBS with Triton X-100 0.1\%]) for 1 hour at RT. Primary antibodies listed in Supplemental Table 10 were applied when stated in the indicated concentrations and incubated overnight at $4^{\circ} \mathrm{C}$ in a humid chamber. Appropriate secondary antibodies (Alexa Fluor dyes from Molecular Probes) were used at a concentration of 1:500 and incubated RT during 3 hours, followed by washing steps, a labeling step with phalloidin-Alexa Fluor 647 (to stain F-actin) or DAPI (to stain nuclei) when indicated. Slides were mounted using Prolong Gold antifade mounting medium or Fluoromount mounting medium, and they were visualized in a Leica TCS SP5 confocal laser microscope using a HCX PLAN APO CS 63×/1.4 oil immersion, HCX PLAN APO CS $40 \times / 1.4$ oil immersion, or HCX PLAN APO CS $20 \times / 1.4$ objective.

For immunofluorescence of cells in culture, cells were grown onto glass coverslips and fixed in PFA 4\% for 15 minutes RT or ice-cold methanol for 5 minutes at $-20^{\circ} \mathrm{C}$ (when indicated); they were then washed in PBS. For neurosphere immunostaining, incipient neurospheres were generated during 2 days in vitro by clonal dilution of primary NPs. Neurospheres were placed onto matrigel-coated glass coverslips (Corning) for 5 minutes and fixed immediately with PFA 4\%. Further details about the antibodies used in this study are included in Supplemental Table 10.

TEM. Cells were grown on P6MW dishes (Corning) and fixed with glutaraldehyde 4\% (MilliporeSigma, G5882). Fixed cells were washed $3 \times$ in $0.1 \mathrm{M}$ cacodylate buffer (MilliporeSigma); they were then postfixed in $1 \%$ osmium tetroxide $/ 1.5 \%$ potassium ferrocyanide for 30 minutes. Cells were then washed $3 \times$ and incubated in 1\% aqueous uranyl acetate (MilliporeSigma) for 30 minutes, washed, and subsequently dehydrated in grades of alcohol (5 minutes each at 50\%,70\%,95\%, and $2 \times 5$ minutes at $100 \%$ ). Cells were embedded in TAAB Epon (Marivac Canada Inc.) and polymerized at $60^{\circ} \mathrm{C}$ for 72 hours. After polymerization, $1 \mathrm{~mm}$ squares of the embedded monolayer were glued onto an empty Epon block for sectioning.

For tissue TEM, E14.5 embryonic brains were dissected and fixed immediately in $2 \%$ glutaraldehyde (MilliporeSigma, G5882) 4\% paraformaldehyde (Electron Microscopy Sciences, 50-980-487) in 0.4M HEPES buffer for 2 hours at RT. Entire embryonic brains were then postfixed with 1\% osmium tetroxide $\left(\mathrm{OsO}_{4}\right) / 1.5 \%$ potassium ferrocyanide $\left(\mathrm{KFeCN}_{6}\right)$ for 1 hour. Then brains were washed in $0.4 \mathrm{M}$ HEPES $3 \times$ and incubated in $1 \%$ aqueous uranyl acetate for 1 hour, followed by 2 washes in $0.4 \mathrm{M}$ HEPES and subsequent dehydration in grades of alcohol (10 minutes each at 50\%, 70\%, 90\%, and $2 \times 10$ minutes at $100 \%$ ). The samples were then infiltrated for 30 minutes in a 1:1 mixture of propylene oxide and TAAB Epon (Marivac Canada Inc.). The samples were embedded in drops of TAAB Epon (Electron Microscopy Sciences) and polymerized in molds at $60^{\circ} \mathrm{C}$ for at least 72 hours. Ultrathin sections of $40 \mathrm{~nm}$ were cut on a Reichert Ultracut-S microtome, placed onto copper TEM grids, stained with uranyl acetate, and examined in a Tecnai G2 spirit transmission electron microscope equipped with a lantane hexaboride (LaB6) filament and a TemCam-F416 $(4 \mathrm{k} \times 4 \mathrm{k})$ camera with CMOS technology.

Cell culture and time-lapse microscopy. Primary MEFs were isolated from E14.5 mouse embryos, dissociated by trypsinization, and cultured in DMEM with high glucose (MilliporeSigma, D6429) supplemented with 10\% FBS (MilliporeSigma), glutamax supplement (Thermo Fisher Scientific, 35050038), and 0.1\% gentamycin (Thermo Fisher Scientific). All experiments were performed with primary cultures of passage 0 (at $\mathrm{P} 0$ ).

For time-lapse microscopy, MEFs were seeded in IBIDI 8 well $\mu$-slide chambers (ibidi, 80826) at a medium confluency and nuclei stained for 30 minutes with SiR-DNA kit (Spirochrome) following manufacturer's instructions. Micrographs in time frames of 10 minutes were acquired during 24 hours 
in a Deltavision RT imaging system (Olympus IX70/71, Applied Precision) equipped with a Plan apochromatic $20 \times / 1.42$ N.A. objective and maintained at $37^{\circ} \mathrm{C}$ in a humidified $\mathrm{CO}_{2}$ chamber. The resulting videos were processed and analyzed with ImageJ software (NIH).

Primary NPs were obtained from E14.5 embryos. Briefly, E14.5 embryonic brains were dissected under aseptic conditions; neocortices from dorsal telencephalon were separated and digested with papain (Worthington, LS00310). Papain was activated in an activation solution consisting of $5.5 \mathrm{mM}$ L-cysteine (MilliporeSigma, C8277) and 1.1 mM EDTA (MilliporeSigma, E6511) in EBSS (Thermo Fisher Scientific, 24010-043) following manufacturer's instructions, filtered through a $0.22 \mu \mathrm{M}$ filter and used at a concentration of $12 \mathrm{U} / \mathrm{mL}$ ( $1 \mathrm{~mL} / 2$ hemicortices) together with DNAse I (Thermo Fisher Scientific) for 30 minutes at $37^{\circ} \mathrm{C}$. NPs obtained were pelleted in DMEM/Hams F-12 (Thermo Fisher Scientific), and activation solution was removed completely. Primary NPs were cultured in suspension in a medium containing DMEM/Hams F-12 (Thermo Fisher Scientific, 11320033), 5 mM HEPES, 1 mM sodium pyruvate, $2 \mathrm{mM}$ L-glutamine, N2 supplement (Thermo Fisher Scientific, 17502048), B27 supplement (Thermo Fisher Scientific, 17504044), $0.7 \mathrm{U} / \mathrm{mL}$ heparin sodium salt (MilliporeSigma, H3149), $20 \mathrm{ng} / \mathrm{mL}$ mouse EGF (Thermo Fisher Scientific, PMG8044), 20 ng/mL mouse FGF2 (Thermo Fisher Scientific, PMG0034), and penicillin/streptomycin (Thermo Fisher Scientific, 15140148). When needed, neurospheres were dissociated with accutase for 5 minutes at $37^{\circ} \mathrm{C}$ prior to experiment plating.

Limiting dilution assays. Limited dilution assays were performed as previously described (42). Briefly, NP cultures were plated at density of 100 cells $/ \mathrm{mL}$ and incubated for 48 hours. Neurospheres formed were dissociated and plated in NP medium in 96-well plates at different cellular densities (100, 50, 20, and 10 cells per well). One week later, neurosphere formation was assessed, and wells in which there was at least 1 neurosphere were considered positive. Data in the corresponding representations indicate the fraction of cells with ability to generate cultures with new neurospheres. Graphs were obtained using the ELDA software (42) that processes the data obtained in each experimental condition to the limiting dilution model. In these graphs, the slopes of the depicted solid lines correspond to the fraction of cells with the ability to generate new spheres cultures. A lower slope value indicates a lower fraction of cells with capacity to generate new neurospheres. Dotted lines represent the 95\%CI.

Flow cytometry and metaphase spreads. Flow cytometry analysis of DNA content was performed by fixing cells with cold $70 \%$ ethanol followed by staining with $10 \mu \mathrm{g} / \mathrm{mL}$ propidium iodide (MilliporeSigma). Data acquisition was performed with a Fortessa LSR analyzer (BD Biosciences).

For metaphase spreads, cultured cells were exposed to colcemid (10 $\mu \mathrm{g} / \mathrm{mL}$; Roche, 295892) for 6 hours and pelleted, and individual cell suspension was hypotonically swollen in $50 \mathrm{~mL}$ of $75 \mathrm{mM} \mathrm{KCl}$ at $37^{\circ} \mathrm{C}$ for $20-30$ minutes. Hypotonic treatment was stopped by gentle addition of $1 \mathrm{~mL}$ of Carnoy's solution (75\% pure methanol, 25\% glacial acetic acid); cells were then spun down and fixed twice with Carnoy's solution for 20-30 minutes at RT. After fixation, cells were dropped from a $1 \mathrm{~m}$ height onto clean, prewarmed glass slides. Cells were dried overnight at $70^{\circ} \mathrm{C}$ and stained with Giemsa (MilliporeSigma) afterward, following standard procedures. Images were acquired with a Leica D3000 microscope and a $60 \times$ Plan Apo N (numerical aperture, 1.42) objective. Chromosomes from 100 cells per genotype were counted using ImageJ software (NIH).

Three-dimensional immunofluorescence of E14.5 embryos (FLASH). Whole E14.5 embryos were processed for FLASH (43) by replacing Borate-SDS with a solution of 3 (dimethyl[tetradecyl]azaniumyl)propane-1-sulfonate detergent $(80 \mathrm{~g} / \mathrm{L})$, boric acid $(200 \mathrm{mM})$, and urea $(250 \mathrm{~g} / \mathrm{L})(44)$. Immunofluorescence was performed as stated above using the antibodies indicated in Supplemental Table 10. Images were acquired with a light sheet microscope (LAvision Ultramicroscope II). Selection Plane Illumination Microscopy (SPIM) image 3D reconstruction and surfaces rendering for positive staining was performed with Imaris v9 software (Bitplane).

RNAseq analysis. Total RNA derived from E11.5 and E14.5 embryonic neocortices and cultured neurospheres was isolated using mirVana kit (Thermo Fisher Scientific), and sample RNA Integrity was assayed on an Agilent 2100 Bioanalyzer. Sequencing libraries were prepared with the QuantSeq 3' mRNA-Seq Library Prep Kit (FWD) for Illumina (Lexogen, catalog 015) by following manufacturer instructions. Library generation was initiated by reverse transcription with oligo(dT) priming, and a second strand synthesis was performed from random primers by a DNA polymerase. Primers from both steps contain Illumina-compatible sequences. cDNA libraries were purified, applied to an Illumina flow cell for cluster generation, and sequenced on an Illumina instrument (see below) by following manufacturer's protocols. Read adapters and polyA tails were removed following the Lexogen recommendations. Processed reads were analyzed 
with the nextpresso pipeline (45), as follows: sequencing quality was checked with FastQC v0.11.7 (http:// www.bioinformatics.babraham.ac.uk/projects/fastqc/). Reads were aligned to the mouse reference genome (GRCm38) with TopHat-2.0.10 using Bowtie 1.0.0 and Samtools 0.1.19 (--library-type fr-secondstrand in TopHat), allowing 3 mismatches and 20 multihits. Read counts were obtained with HTSeq-count v0.6.1 (--stranded=yes), using the mouse gene annotation from GENCODE (gencode.vM20.GRCm38.Ensemb195). Differential expression was performed with DESeq2, using a 0.05 FDR. Enrichr (46) and DAVID (47) were used for gene set enrichment analysis of differentially expressed genes. GSEAPreranked was used to perform gene set enrichment analysis for several gene signatures on a preranked gene list, setting 1000 gene set permutations. Only those gene sets with significant enrichment levels (FDR $q<0.25$ ) were considered. Access to RNAseq data are provided from the Gene Expression Omnibus (GEO GSE14749).

Statistics. Statistics was performed using Prism software (GraphPad Software) or Microsoft Excel. All statistical tests of comparative data were done using a Mann-Whitney $U$ test or an unpaired, 2-tailed Student's $t$ test with Welch's correction, or 1-way ANOVA with Tukey's multiple-comparison test when appropriate. Data are expressed as the mean of at least 3 independent experiments $\pm \mathrm{SEM}$; a $P$ value of less than 0.05 considered statistically significant.

Study approval. Mice were housed in a pathogen-free animal facility at the CNIO following the animal care standards of the institution. The animals were observed on a daily basis, and sick mice were humanely euthanized in accordance with the Guidelines for Humane End-points for Animals Used in Biomedical Research (Directive 2010/63/EU of the European Parliament and Council and the Recommendation 2007/526/CE of the European Commission). All animal protocols were approved by the committee for animal care and research of the Instituto de Salud Carlos III/Comunidad de Madrid (Madrid, Spain).

\section{Author contributions}

JGM performed most of the experiments. AWC, DMA, LRLS, JGJ, AM, and AP participated in the analysis of cellular phenotypes and analysis of embryo abnormalities. JA and AB performed the whole-mount immunofluorescence studies. DM, JG, and MPM helped with fluorescent microscopy and JB contributed to TEM studies. OGC helped with the bioinformatics analysis. SO contributed to the injection of mouse embryos. MM designed the project, and JGM and MM wrote the manuscript. All the authors contributed to the analysis of the data.

\section{Acknowledgments}

We thank Pilar Redondo and Carmen García Martín (CNIO) for technical assistance and acquisition of TEM micrographs, and the electron microscopy service at Centro de Investigaciones Biológicas (CIB, Madrid) for TEM ultrathin sections preparation. We also thank the Mouse Genome Editing Unit (CNIO) for zygote microinjections for the generation of the CRISPR-edited mutant mice. JGM and DMA received predoctoral contracts from the Ministry of Education of Spain (FPI grants). AWC was supported by Fondation pour la Recherche Médicale and European Molecular Biology Organization (EMBO). JGJ received a short fellowship from EMBO. This work was supported by a grant from the European Commission Seventh Framework Programme (ERA-NET NEURON8-Full-815-094), grants from AEI-MICIU/FEDER (RTI2018-095582-B-I00 and RED2018-102723-T), Worldwide Cancer Research and the AECC (WCR20155), and the iLUNG program from the Comunidad de Madrid (B2017/BMD-3884). CNIO is a Severo Ochoa Center of Excellence (AEI-MICIU CEX2019-000891-S).

Address correspondence to: Marcos Malumbres, Centro Nacional de Investigaciones Oncológicas (CNIO), Melchor Fernández Almagro 3, E-28029 Madrid, Spain. Phone: 34.917328000; Email: malumbres@cnio.es.

1. Megraw TL, et al. Cdk5rap2 exposes the centrosomal root of microcephaly syndromes. Trends Cell Biol. 2011;21(8):470-480.

2. Jayaraman D, et al. Microcephaly proteins Wdr62 and Aspm define a mother centriole complex regulating centriole biogenesis, apical complex, and cell fate. Neuron. 2016;92(4):813-828.

3. Thornton GK, Woods CG. Primary microcephaly: do all roads lead to Rome? Trends Genet. 2009;25(11):501-510.

4. Saade M, et al. A centrosomal view of CNS growth. Development. 2018;145(21):dev170613.

5. Bettencourt-Dias M, Glover DM. Centrosome biogenesis and function: centrosomics brings new understanding. Nat Rev Mol Cell Biol. 2007;8(6):451-463.

6. Bettencourt-Dias M, et al. SAK/PLK4 is required for centriole duplication and flagella development. Curr Biol. 2005;15(24):2199-2207. 
7. Habedanck R, et al. The Polo kinase Plk4 functions in centriole duplication. Nat Cell Biol. 2005;7(11):1140-1146.

8. Hung LY, et al. Protein 4.1 R-135 interacts with a novel centrosomal protein (CPAP) which is associated with the gamma-tubulin complex. Mol Cell Biol. 2000;20(20):7813-7825.

9. Leidel S, et al. SAS-6 defines a protein family required for centrosome duplication in C. elegans and in human cells. $\mathrm{Nat} C e l l$ Biol. 2005;7(2):115-125.

10. Ohta T, et al. Characterization of Cep135, a novel coiled-coil centrosomal protein involved in microtubule organization in mammalian cells. J Cell Biol. 2002;156(1):87-99.

11. Dobbelaere J, et al. A genome-wide RNAi screen to dissect centriole duplication and centrosome maturation in Drosophila. PLoS Biol. 2008;6(9):e224.

12. Carvalho-Santos Z, et al. BLD10/CEP135 is a microtubule-associated protein that controls the formation of the flagellum central microtubule pair. Dev Cell. 2012;23(2):412-424.

13. Mottier-Pavie V, Megraw TL. Drosophila bld10 is a centriolar protein that regulates centriole, basal body, and motile cilium assembly. Mol Biol Cell. 2009;20(10):2605-2614.

14. Roque H, et al. Drosophila Cep135/Bld10 maintains proper centriole structure but is dispensable for cartwheel formation. J Cell Sci. 2012;125(pt 23):5881-5886.

15. Inanc B, et al. Abnormal centrosomal structure and duplication in Cep135-deficient vertebrate cells. Mol Biol Cell. 2013;24(17):2645-2654.

16. Lin YC, et al. Human microcephaly protein CEP135 binds to hSAS-6 and CPAP, and is required for centriole assembly. EMBO J. 2013;32(8):1141-1154.

17. Hussain MS, et al. A truncating mutation of CEP135 causes primary microcephaly and disturbed centrosomal function. Am J Hum Genet. 2012;90(5):871-878.

18. Bamborschke D, et al. Mutation in CEP135 causing primary microcephaly and subcortical heterotopia. Am JMed Genet $A$. 2020;182(10):2450-2453.

19. Pulvers JN, et al. Mutations in mouse Aspm (abnormal spindle-like microcephaly associated) cause not only microcephaly but also major defects in the germline. Proc Natl Acad Sci U S A. 2010;107(38):16595-16600.

20. Zaqout S, et al. CDK5RAP2 is required to maintain the germ cell pool during embryonic development. Stem Cell Reports. 2017;8(2):198-204.

21. Lizarraga SB, et al. Cdk5rap2 regulates centrosome function and chromosome segregation in neuronal progenitors. Development. 2010;137(11):1907-1917.

22. Nasser H, et al. $C D K 5 R A P 2$ primary microcephaly is associated with hypothalamic, retinal and cochlear developmental defects. J Med Genet. 2020;57(6):389-399.

23. Alfares A, et al. A new association between CDK5RAP2 microcephaly and congenital cataracts. Ann Hum Genet. 2018;82(3):165-170

24. Orosco LA, et al. Loss of Wdfy3 in mice alters cerebral cortical neurogenesis reflecting aspects of the autism pathology. Nat Commun. 2014;5:4692

25. Jayaraman D, et al. The genetics of primary microcephaly. Annu Rev Genomics Hum Genet. 2018;19:177-200.

26. Barbelanne M, Tsang WY. Molecular and cellular basis of autosomal recessive primary microcephaly. Biomed Res Int. 2014;2014:547986.

27. Farooq M, et al. A novel splice site mutation in CEP135 is associated with primary microcephaly in a Pakistani family. $J$ Hum Genet. 2016;61(3):271-273.

28. Carvalho-Santos Z, et al. Stepwise evolution of the centriole-assembly pathway. J Cell Sci. 2010;123(pt 9):1414-1426

29. Gonczy P. Towards a molecular architecture of centriole assembly. Nat Rev Mol Cell Biol. 2012;13(7):425-435.

30. Hilbert M, et al. SAS-6 engineering reveals interdependence between cartwheel and microtubules in determining centriole architecture. Nat Cell Biol. 2016;18(4):393-403.

31. Bazzi H, Anderson KV. Acentriolar mitosis activates a p53-dependent apoptosis pathway in the mouse embryo. Proc Natl Acad Sci U S A. 2014;111(15):E1491-E1500.

32. Insolera R, et al. Cortical neurogenesis in the absence of centrioles. Nat Neurosci. 2014;17(11):1528-1535.

33. McKinley KL, Cheeseman IM. Large-scale analysis of CRISPR/Cas9 cell-cycle knockouts reveals the diversity of p53-dependent responses to cell-cycle defects. Dev Cell. 2017;40(4):405-420.

34. Little JN, Dwyer ND. p53 deletion rescues lethal microcephaly in a mouse model with neural stem cell abscission defects. Hum Mol Genet. 2019;28(3):434-447.

35. Marjanovic M, et al. CEP63 deficiency promotes p53-dependent microcephaly and reveals a role for the centrosome in meiotic recombination. Nat Commun. 2015;6:7676

36. Fish JL, et al. Aspm specifically maintains symmetric proliferative divisions of neuroepithelial cells. Proc Natl Acad Sci U S A. 2006;103(27):10438-10443.

37. Gai M, et al. ASPM and CITK regulate spindle orientation by affecting the dynamics of astral microtubules. EMBO Rep. 2016;17(10):1396-1409.

38. Yu TW, et al. Mutations in WDR62, encoding a centrosome-associated protein, cause microcephaly with simplified gyri and abnormal cortical architecture. Nat Genet. 2010;42(11):1015-1020.

39. Oegema R, et al. Subcortical heterotopic gray matter brain malformations: classification study of 107 individuals. Neurology. 2019;93(14):e1360-e1373.

40. Stoner R, et al. Patches of disorganization in the neocortex of children with autism. N Engl J Med. 2014;370(13):1209-1219.

41. Henao-Mejia J, et al. Generation of genetically modified mice using the CRISPR-Cas9 genome-editing system. Cold Spring Harb Protoc. 2016;2016(2):pdb prot090704.

42. Hu Y, Smyth GK. ELDA: extreme limiting dilution analysis for comparing depleted and enriched populations in stem cell and other assays. J Immunol Methods. 2009;347(1-2):70-78.

43. Messal HA, et al. Tissue curvature and apicobasal mechanical tension imbalance instruct cancer morphogenesis. Nature. 2019;566(7742):126-130. 
44. Tedeschi A, et al. Cep55 promotes cytokinesis of neural progenitors but is dispensable for most mammalian cell divisions. Nat Commun. 2020;11(1):1746.

45. Graña O, et al. Nextpresso: next generation sequencing expression analysis pipeline. Curr Bioinform. 2018;13(6):583-591.

46. Kuleshov MV, et al. Enrichr: a comprehensive gene set enrichment analysis web server 2016 update. Nucleic Acids Res. 2016;44(w1):W90-W97.

47. Huang DW, et al. Systematic and integrative analysis of large gene lists using DAVID bioinformatics resources. Nat Protoc 2009;4(1):44-57. 